Nouvelles perspectives en sciences sociales

Revue internationale de systémique complexe et d'études relationnelles

\title{
Les facteurs cognitifs de l'objectivation du territoire : relations épistémiques et représentations dans la gestion de l'eau en Camargue
}

\section{Aurélien Allouche}

Volume 10, numéro 1, novembre 2014

Sur le thème du territoire

URI : https://id.erudit.org/iderudit/1028437ar

DOI : https://doi.org/10.7202/1028437ar

Aller au sommaire du numéro

Éditeur(s)

Prise de parole

ISSN

1712-8307 (imprimé)

1918-7475 (numérique)

Découvrir la revue

Citer cet article

Allouche, A. (2014). Les facteurs cognitifs de l'objectivation du territoire :

relations épistémiques et représentations dans la gestion de l'eau en

Camargue. Nouvelles perspectives en sciences sociales, 10(1), 59-113.

https://doi.org/10.7202/1028437ar
Résumé de l'article

Cet article aborde la problématique du territoire selon une perspective émergente en sociologie, l'étude des dimensions cognitives et épistémiques de la gouvernance et de la démocratie participative. Il se concentre sur le travail collectif d'objectivation du territoire rendu nécessaire par sa gestion collective pour interroger l'intégration des représentations des habitants et des savoirs profanes au processus de décision. En considérant les propriétés épistémiques et cognitives de l'objectivation, l'auteur identifie deux processus limitant l'intégration des connaissances profanes et des représentations locales à la définition du territoire de projet : la dynamique des relations épistémiques et les modalités d'apprentissage organisationnel sous forme de règle de gestion. L'article prend pour cas d'étude la gestion collective de l'eau en Camargue (France, Bouches-du-Rhône) et s'appuie sur l'observation sociologique d'une commission participative réalisée de 2009 à 2012. 


\title{
Les facteurs cognitifs de l'objectivation du territoire : relations épistémiques et représentations dans la gestion de l'eau en Camargue
}

\author{
Aurélien Allouche \\ Lames, UMR 7305, \\ Université d'Aix-Marseille - CNRS
}

\section{Introduction}

T 'aménagement et la gestion de milieux urbains et d'espaces naturels sont régulièrement le lieu de confrontation de différentes représentations et conceptions du territoire. Ces confrontations, qui vont parfois jusqu'au conflit ouvert, ont en commun de mettre en lice un "territoire de projet", correspondant à une vision planifiée défendue par un groupe décisionnaire ou gestionnaire, et des territoires vécus, pratiqués et appropriés par différents individus et groupes sociaux. Ces nombreux conflits, objets de longue date de disciplines telles que la sociologie, l'histoire, les sciences politiques ou la géographie, sont le plus souvent doublés d'une revendication pour la reconnaissance de savoirs et de connaissances "profanes».

L'auteur remercie Michel Favory, professeur à Sciences Po Bordeaux, pour sa relecture avisée, ainsi que Laurence Nicolas, Anthropologue à RESSOURCE, pour ses conseils précieux. 
Certes, la reconnaissance des représentations et des connaissances des habitants et des usagers n'est un enjeu ni spécifique ni exclusif des conflits liés à l'aménagement et à la gestion des territoires. Toutefois, dans ce cadre de réflexion, la confrontation des différents types de connaissances prend une importance toute particulière en joignant au territoire vécu de l'habitant et au territoire de projet des gestionnaires un troisième terme, celui du territoire objectivé. De surcroît, différentes politiques publiques ${ }^{2}$ portent, plus ou moins explicitement, l'ambition de fonder une gestion participative du bien public sur des processus d'objectivation des connaissances.

La recherche en sciences sociales contribue, pour sa part, à développer une conception de la décentration des représentations territoriales dans des connaissances objectivées, c'est-à-dire une conception de la façon dont les acteurs aboutissent, par-delà leurs représentations personnelles, à une définition intersubjective de qui fait territoire. Dans une logique habermassienne, l'objectivation du territoire à travers des connaissances dépassant les interprétations subjectives et les représentations sociales est pensée comme pouvant fournir un espace de ralliement aux parties prenantes d'un conflit. D'autres traditions de pensée fournissent également des lignes d'analyse fécondes pour aborder les processus d'objectivation des connaissances et des percepts susceptibles d'entrer dans la gestion collective d'un territoire. La phénoménologie sociale d'Alfred Schutz a conduit à traiter prioritairement les processus de socialisation à travers lesquels des acteurs aboutissent à des cadres d'interprétation partagés ${ }^{3}$. De même, différents travaux issus de l'interactionnisme symbolique ainsi que de l'ethnométhodologie ont analysé la construction de connaissances d'arrière-plan que les acteurs mobilisent pour

2 Par exemple, dans le cas de la France, on peut citer la loi Barnier de 1995, jusqu'à l'institutionnalisation du débat public par la loi de démocratie de proximité adoptée en février 2002 et l'instauration des Commissions nationales du débat public. La convention européenne d'Aarhus de 1998 semble porter un présupposé similaire, de même que la directive européenne «Inspire» de 2007.

3 Alfred Schutz, Le chercheur et le quotidien: phénoménologie des sciences sociales, Paris, Méridiens Klincksieck, 1987. 
aboutir à une compréhension partagée du monde ${ }^{4}$. Différents courants pragmatistes permettent de décrire l'objectivation des connaissances et des percepts comme une activité sociale continue ${ }^{5}$. Héritant partiellement de ces traditions de pensée, la sociologie dite de la "traduction ${ }^{6}$ " a plus spécifiquement porté son attention sur les pratiques et les dispositifs grâce auxquels les acteurs tendent à produire des états du monde qui seront traités comme "objectifs", car ne pouvant plus être rattachés à un énonciateur individuel, mais prenant sens dans un réseau de relations entre humains et non-humains.

Selon ce rapide panorama, un premier ensemble de travaux, plus ou moins hétérogène, suggère que le «territoire de projet» et les "territoires vécus» peuvent se concilier, voire s'hybrider, dans la gestion participative grâce au processus d'objectivation

$4 \quad$ Peter Berger et Thomas Luckmann, La construction sociale de la Réalité, Paris, Armand Colin, 1996; Aaron Cicourel, Cognitive Sociology: Language and Meaning in Social Interaction, New York, Free Press, 1974; Harold Garfinkel, Studies in Ethnomethodology, Englewood Cliffs (NJ), Prentice-Hall, 1967; Anselm Strauss, Miroirs et masques. Une introduction à l'interactionnisme, Paris, Métailié, 1992.

5 Les familles de pensées sont ici très variées, depuis les figures tutélaires de William James et de John Dewey, jusqu'à des approches prioritairement ontologiques, telles que celle de John Searle (La construction de la réalité sociale, Paris, Gallimard, 1998), et à des constructivismes interactionnistes à l'image des travaux de Paul Watzlawick (La réalité de la réalité, Paris, Seuil, 1978). Francis Chateauraynaud a récemment présenté une synthèse des différents courants pragmatistes dans le cadre de la sociologie des controverses (Argumenter dans un champ de forces. Essai de balistique sociologique, Paris, Pétra, 2011). De nombreux auteurs s'inscrivent peu ou prou dans l'héritage pragmatiste, ainsi qu'en témoignent Daniel Cefaï et Isaac Joseph (L'Héritage du pragmatisme. Conflits d'urbanité et épreuves de civisme, La Tour-d'Aigues, Éditions de l'Aube, 2002) ou encore l'essai mené par Mohamed Nachi de regrouper les sociologies de Luc Boltanski, Laurent Thévenot, Bruno Latour et de Michel Callon (Introduction à la sociologie pragmatique. Vers un nouveau "style» sociologique?, Paris, Armand Colin, 2006).

6 Madeleine Akrich, Michel Callon et Bruno Latour (dir.), Sociologie de la traduction. Textes fondateurs, Paris, Presse des Mines, 2006; Michel Callon, "Éléments pour une sociologie de la traduction: la domestication des coquilles St-Jacques et des marins pêcheurs dans la baie de St. Brieuc", L'Année Sociologique, vol. 36, 1986, p. 169-208; Bruno Latour et Steve Woolgar, La vie de laboratoire, la production des faits scientifiques, Paris, La Découverte, 1988. 
du territoire. La psychologie sociale et la sociologie des représentations sociales ${ }^{7}$ confortent cette lecture. En particulier, elles préviennent de toute dérive qui consisterait à penser l'objectivation comme le fait des seuls acteurs experts (scientifiques, notamment) et comme analysable à partir d'une stricte opposition entre connaissances formelles et représentations sociales. La posture de recherche empruntée par la théorie des représentations sociales, en refusant de réduire les représentations des acteurs à des déformations des connaissances scientifiques, conduit à traiter l'objectivation comme un processus par lequel un groupe "naturalise» un objet, en structurant la représentation autour d'un «noyau figuratif ${ }^{8}$ ». Cette conception a été prolongée par la logique naturelle de Jean-Blaise Grize' ${ }^{9}$, notamment en sociologie des représentations sociales ${ }^{10}$. L'objectivation peut alors être comprise comme l'ancrage durable, au cours de controverses et de débats sociaux, de notions ou de raisonnements qui finissent par être tenus par les acteurs pour acquis, pour «naturels» ou «évidents", et constituer ainsi une «matrice culturelle d'interprétation ». Ces éléments, qui ne font plus débat, peuvent à leur tour être mobilisés pour discuter, pour expliquer ou pour rendre concrets de nouveaux contenus symboliques sur lesquels des acteurs s'opposent.

La ligne d'analyse pragmatiste et la sociologie des représentations sociales nous incitent à adopter une définition minimaliste de l'objectivation. Nous comprendrons dans ce qui suit l'objectivation

$7 \quad$ Jean-Claude Abric, Pratiques sociales et représentations, Paris, Presses universitaires de France, 1994; Christian Guimelli, Structures et transformations des représentations sociales, Lausanne, Delachaux et Niestlé, 1994; Pierre Vergès, "Représentations sociales partagées, périphériques, indifférentes, d'une minorité", Les Cahiers internationaux de psychologie sociale, $\mathrm{n}^{\circ} 28$, 1995, p. 77-95.

8 Christian Guimelli, La pensée sociale, Paris, Presses universitaires de France, 1999 , p. 65.

9 Jean-Blaise Grize, Logique et langage, Paris, Ophrys, 1990.

10 Jean-Blaise Grize, Pierre Vergès et Ahmed Silem, Les salariés face aux nouvelles technologies, Paris, Éditions du CNRS, 1987; Pierre Vergès, «Représentations sociales de l'économie : une forme de connaissance", dans Denise Jodelet (dir.), Les représentations sociales, Paris, Presses universitaires de France, 1989, p. 387-405. 
comme l'institution collective de cognitions d'arrière-plan mutuelles. Par "cognitions d'arrière-plan" nous entendons des raisonnements, des notions, des connaissances qui sont tenus pour évidents et naturels par les acteurs et que ceux-ci utilisent pour interpréter une situation ou prendre une décision. Ces cognitions sont dites «mutuelles" lorsque chaque acteur tient pour acquis que les autres acteurs possèdent ces cognitions et tiennent, à leur tour, pour acquis que les premiers acteurs sont dans la même disposition vis-à-vis de ces cognitions. La condition de mutualité est élémentaire, mais indispensable pour fonder des interprétations et des décisions collectives à partir de cognitions. Enfin, nous traitons de l'objectivation comme d'un processus qui débouche sur l'institution d'une cognition. Les modalités d'institution sont très variées, pouvant se comprendre comme la formation d'une règle de décision, l'inscription dans une mémoire externalisée ${ }^{11}$, la stabilisation d'un réseau humains/non-humains, ou encore l'évolution d'un système technique ${ }^{12}$. Les cas de figure sont très divers et renvoient à autant de champs d'études différents, c'est pourquoi nous choisissons de ne pas «fermer» cette définition. Transversalement à la diversité des cas, une condition élémentaire paraît être qu'une cognition partagée est traduite sous une forme qui la rend accessible à des acteurs futurs, les dispensant de devoir reproduire le processus d'apprentissage collectif (et d'innovation collective).

Cette définition, en se centrant sur les modalités de compréhension réciproque des acteurs et de décision collective, invite à concentrer notre questionnement sur une dimension particulière du processus d'objectivation, les échanges épistémiques, c'est-àdire les échanges entre acteurs portant sur des connaissances et sur leurs évaluations. Dans la mesure où autour de processus d'objectivation se rencontrent les territoires vécus et le territoire de projet, les échanges épistémiques, lorsqu'ils s’opèrent dans la

11 Aaron Cicourel, op. cit.

12 Anthony Giddens, Les conséquences de la modernité, Paris, L'Harmattan, 1994; Alain Gras, Les macro-systèmes techniques, Paris, Presses universitaires de France, 1997. 
gestion collective d'un espace ou d'une ressource, peuvent-ils contribuer à donner au territoire une consistance sociale spécifique?

Ces remarques préalables incitent à étudier les propriétés selon lesquelles les connaissances vernaculaires ou "techniciennes", profanes ou expertes, permettent ou inversement empêchent la socialisation d'une conception commune du territoire ainsi que l'articulation de valeurs, de représentations et d'intérêts hétérogènes dans la gestion collective d'un territoire et dans son aménagement concerté.

On étudiera trois dimensions essentielles des échanges concourant à l'objectivation du territoire dans la gestion participative, en prenant pour fil directeur le lien entre les modes de socialisation des données et des connaissances et la participation des usagers à la prise de décision. En premier lieu, on abordera la nature des relations que les acteurs établissent entre eux dès lors que le processus d'objectivation du territoire rend nécessaire une division du travail cognitif ${ }^{13}$, c'est-à-dire dès lors que ces acteurs sont contraints de s'en remettre à d'autres pour interpréter une donnée ou une connaissance qu'ils n'ont pas eux-mêmes produite. Ce point conduira à examiner, dans un deuxième temps, la capacité de la gestion participative à intégrer les éléments constitutifs des connaissances vernaculaires, qu'il s'agisse d'indices perceptifs ou de structures de raisonnement. Toutefois, l'étude des relations épistémiques et des critères d'établissement d'une connaissance vernaculaire est insuffisante pour circonscrire le processus d'objectivation. Celui-ci suppose que les connaissances partagées soient, à leur tour, instituées dans une organisation collective et traduites dans des règles de décision qui s'imposeront «naturellement» à de futurs acteurs. Aussi, dans un troisième temps, étudiera-t-on la dimension organisationnelle de l'objectivation territoriale à travers la formation et l'application de règles collectives de décision.

Ce questionnement s'appuiera sur une étude de cas, menée de 2009 à 2012, qui a consisté dans le suivi d'une commission

13 Philip Kitcher, «The Division of Cognitive Labor», Journal of Philosophy, vol. 87, $\mathrm{n}^{\circ} 1,1990$, p. 5-22. 
participative de gestion de l'eau en Camargue (France, Bouchesdu-Rhône ${ }^{14}$. Avant d'aborder ces différents éléments d'analyse, il convient donc de présenter le contexte spécifique de l'étude de cas.

\section{La gestion concertée de l'eau en Camargue, source de territoires, de projets et de connaissances}

\subsection{Situation générale d'un cas d'école}

Les Parcs Naturels Régionaux ont été institués en France, ainsi que Romain Lajarge ${ }^{15}$ l'a montré, tout à la fois en instruments d'aménagement du territoire, en entités territoriales à proprement parler, et en outils de territorialisation des espaces sur la base d'un projet partagé. Les Parcs Naturels Régionaux sont ainsi devenus au cours du temps de véritables vecteurs de territorialisation des politiques publiques. Le Parc Naturel Régional de Camargue (dénommé "PNRC» ci-après) s’inscrit pleinement dans cette logique. Si ses actions, comme sa charte, obéissent à un objectif d'aménagement du territoire, le PNRC est également perçu et vécu comme une entité territoriale, comme en atteste, lors de l'élargissement de son périmètre, en 2011, à l'occasion de la révision de sa charte, les vives controverses ainsi provoquées par la question de l'intégration de l'agglomération de Port-SaintLouis-du-Rhône au territoire de la Camargue alors qu'elle est située en rive gauche du grand Rhône. Mais c'est également un

$14 \quad$ La méthode employée a consisté en un suivi direct des réunions de participation, la transcription intégrale des réunions faisant ensuite l'objet d'une analyse discursive spécifique en recourant aux méthodes de l'analyse conversationnelle et de la sociologie de l'argumentation. Ces analyses ont été complétées par des entretiens informels avec les membres de la commission exécutive de l'eau (CEDE) ainsi que des entretiens semi-directifs. Enfin, l'analyse s'est appuyée sur l'étude des archives de la CEDE. Nous remercions le Parc Naturel Régional de Camargue d'avoir généreusement mis à notre disposition ces archives et d'avoir permis l'observation continue de la CEDE.

15 Romain Lajarge, "Territorialisation(s) et parcs naturels régionaux", dans Alain Faure et Emmanuel Négrier (dir.), Les politiques publiques à l'épreuve de l'action locale. Critiques de la territorialisation, Paris, L'Harmattan, 2007, p. 69-78. 
«territoire de projet» dans la mesure où la principale mission du PNRC est l'animation d'un territoire autour d'objectifs, de valeurs et d'actions définis en concertation dans sa charte.

$\mathrm{Au}$ sein de ce projet, la gestion de l'eau occupe une place essentielle, car elle conditionne très largement les composantes économique, écologique et sociale de ce territoire. En concentrant son attention sur la gestion participative de l'eau, on est appelé à traiter de la rencontre de trois entités : le territoire objectivé (dans la mesure où cette gestion repose sur la mobilisation constante d'une qualification scientifique et technique du milieu), le territoire vécu et pratiqué des habitants et des usagers participant à la gestion, enfin, le territoire de projet que les principaux gestionnaires (PNRC, Réserve Naturelle de Camargue, communes d'Arles et des Saintes-Maries-de-la-Mer) portent au travers des décisions prises dans la gestion de l'eau.

On traitera ici de la Camargue en tant qu'elle est comprise dans son acception stricte, à savoir la région délimitée par les deux bras du Rhône aval et par la mer Méditerranée, ce qui est parfois également appelé «l'Île de Camargue» (voir la figure 1). Cet espace s'est constitué dans sa physionomie actuelle par l'endiguement du Rhône et par des travaux d'assèchement successifs qui finirent par convertir cette terre marécageuse en un espace agricole important, où la riziculture prend son essor à partir des années 1950 pour devenir progressivement la culture dominante. Grâce à l'immersion des parcelles qu'elle nécessite, la culture du riz présente l'avantage de s'affranchir de la forte salinité de la terre. La Camargue s'est ainsi rapidement poldérisée. On estime que $55 \%$ de la surface de l'île de Camargue sont poldérisés : 400 Mm3 d'eau sont pompés au Rhône pour les besoins de la riziculture, l'eau de drainage est alors partiellement renvoyée au Rhône par pompage, mais un peu plus de $100 \mathrm{Mm} 3$ d'eau sont acheminés dans l'étang du Vaccarès par des ouvrages gravitaires ${ }^{16}$.

$\overline{16}$ Pierre Heurteaux, «Essai de quantification des termes du bilan hydrique des étangs du système Vaccarès Camargue (France)", Annales de limnologie, vol. 30, n 2,1994, p. 131-144; Philippe Chauvelon et al., "Satellite Remote Sensing and GIS Used to Quantify Water Input for Rice Cultivation (Rhône 
Figure 1:

Carte de situation de la Camargue (île de Camargue)

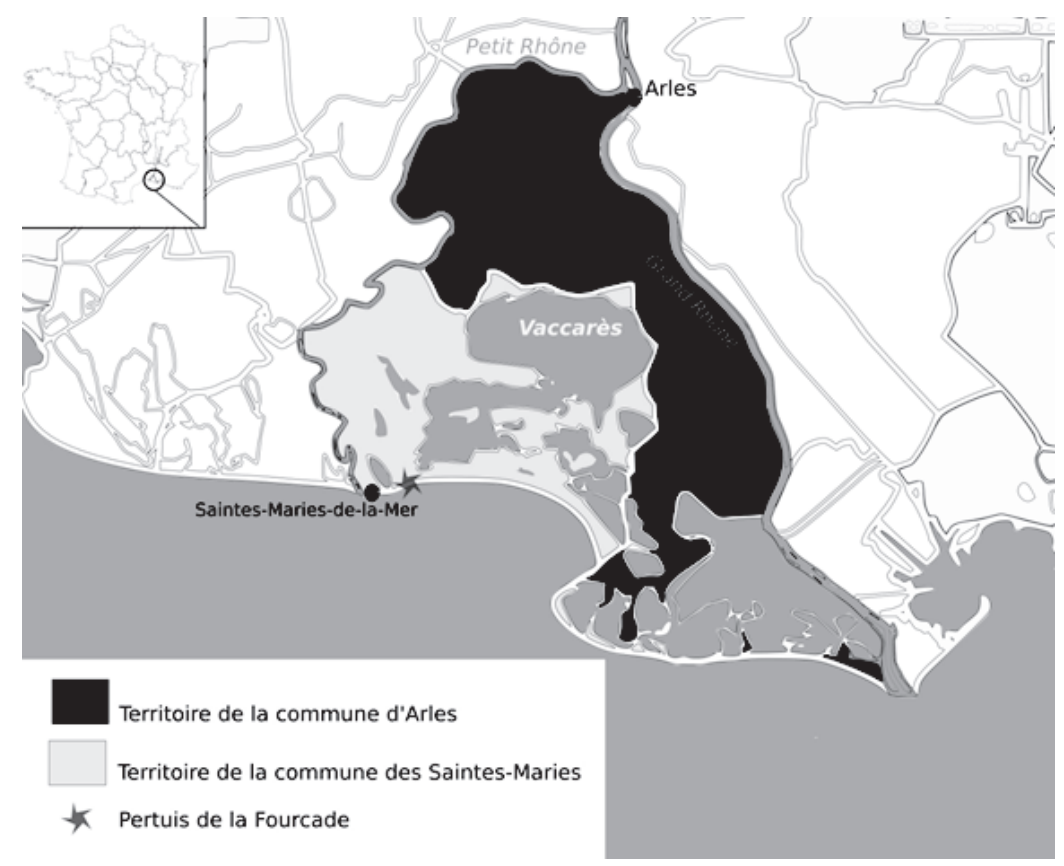

L'équilibre économique de la Camargue, de même que sa morphologie paysagère, ou encore l'état de ses écosystèmes se trouvent de ce fait fortement dépendants de la gestion de l'eau. Cela crée une situation paradoxale, connue et assumée de longue date ${ }^{17}$, où l'équilibre de milieux naturels dépend quasi intégralement d'apports hydrauliques artificiels ${ }^{18}$. Le nœud gordien de la gestion de l'eau, donc de cet équilibre, réside dans la gestion du Vaccarès et spécifiquement de son exutoire à la mer : le pertuis

delta, France) », dans Manfred Owe et al. (dir.), Remote Sensing and Hydrology 2000, Wallingford, IAHS Publication, 2001, p. 446-450.

17 Jacques Béthemont, "Le riz et la mise en valeur de la Camargue", Revue de géographie de Lyon, vol. 37, n 2, 1962, p. 153-206; Bernard Picon, L'espace et le temps en Camargue, Arles, Actes Sud, 1978.

18 Raphäl Mathevet, Camargue incertaine. Sciences, usages et natures, Paris, Buchet Chastel, 2004. 
de la Fourcade, un ouvrage de treize vannes permettant la communication des étangs avec la mer.

La gestion de l'eau en Camargue peut se résumer par la rencontre et le ménagement de deux sous-unités territoriales, le nord de la Camargue et le sud, le premier appartenant principalement à la commune d'Arles, le second à la commune des SaintesMaries-de-la-Mer. L'étang du Vaccarès et les étangs centraux ${ }^{19}$ font office d'une dimension commune à ces deux sous-unités. Les apports d'eau rizicoles proviennent principalement du nord de la Camargue pour être rejetés dans l'étang du Vaccarès. L'ampleur de ces rejets concourt à maintenir cet étang et le système d'étangs centraux à des cotes importantes, créant un risque de débordement du plan d'eau aussi bien sur la rive nord du Vaccarès que sur sa rive sud. La seule possibilité de gérer cette situation consiste en l'actionnement des vannes du pertuis de la Fourcade, lequel se situe sur le territoire de la commune des Saintes-Maries-de-la-Mer.

1.2. L'organisation collective de la gestion de l'eau : structure et acteurs

Une commission, la Commission Exécutive de l'Eau (CEDE), placée sous la présidence de la DDTM ${ }^{20}$ des Bouches-du-Rhône et animée par le PNRC, a pour charge d'organiser la gestion collective de ce pertuis. Nous avons suivi l'activité de la CEDE de 2009 à $2012^{21}$.

La gestion de l'exutoire à la mer doit obéir à des enjeux divers et parfois antagonistes. Si le pertuis de la Fourcade est ouvert à son maximum (treize vannes) en situation «favorable», c'est-àdire en situation de fort vent de secteur nord, la cote de l'étang peut alors être maintenue à un niveau suffisamment bas pour

19 L'ensemble de ces territoires est qualifié de «système Vaccarès» en raison de leur connexion hydraulique.

20 Directions départementales des territoires et de la mer.

21 Le suivi a été réalisé dans le cadre d'un programme de recherche «Eau et Territoire» financé par le Ministère de l'environnement, à l'intérieur du projet "CAMPLAN. Gestion intégrée d'un hydrosystème: Camargue et Plan du Bourg». 
prévenir un risque de débordement. Cet abaissement du niveau d'eau est pénalisant pour les pêcheurs qui travaillent dans les étangs inférieurs du système Vaccarès car, le niveau décroissant plus rapidement à proximité de la mer, la hauteur d'eau n'est pas suffisante pour installer les filets de pêche et réaliser des prises intéressantes. En revanche, l'activité des pêcheurs qui exercent au nord de l'étang du Vaccarès n'est pas incommodée par une ouverture trop importante au sud, car les cotes restent suffisantes pour de bonnes conditions de pêche. Si néanmoins l'ouverture du pertuis conduit à une baisse trop importante du niveau des étangs, ces pêcheurs peuvent être également pénalisés : une déconnexion hydraulique s'opère en effet entre les étangs inférieurs (qui communiquent avec la mer) et les étangs centraux, ne permettant plus aux espèces piscicoles (l'anguille notamment) venant de la mer de peupler la lagune qu'est l'étang du Vaccarès.

Les acteurs naturalistes et gestionnaires (Réserve Naturelle de Camargue et PNRC), responsables du plan de gestion des milieux et participant de manière déterminante au projet de territoire défini par le PNRC, sont également défavorables à un trop grand abaissement des niveaux lorsque cela va à l'encontre de flux biologiques caractéristiques d'un système lagunaire. Les riverains du Vaccarès, les exploitants agricoles et les éleveurs, lorsqu'ils prennent part aux décisions de la Commission Exécutive de l'Eau (CEDE), sont généralement favorables à une maximisation des rejets à la mer dans la mesure où les situations permettant l'évacuation sont rendues rares par l'élévation du niveau marin; la mer est en effet le plus souvent à une cote supérieure à celle des étangs, ce qui rend impossible l'évacuation de ces derniers par le pertuis de la Fourcade ${ }^{22}$. La riziculture non poldérisée, dont les effluents sont en partie responsables de la difficulté à gérer cette situation, a besoin de maintenir le Vaccarès à un niveau suffisamment bas pour permettre le ressuyage et le drainage des terres cultivées puisque les drains et les canaux d'évacuation

$\overline{22}$ Alain Dervieux, «La difficile gestion globale de l'eau en Camargue (France): le Contrat de delta», Vertigo, 2005, [en ligne] http://vertigo.revues.org/2411, site consulté en octobre 2014. 
fonctionnent par gravité et se rejettent dans le Vaccarès. Enfin, la pratique de la chasse dans la partie sud de la Camargue est pénalisée (sur certaines chasses publiques) par un manque d'eau dans les étangs inférieurs, c'est tout au moins la perception qu'en ont certains chasseurs ${ }^{23}$.

\subsection{Comment la question de l'objectivation du territoire se pose dans la gestion concertée de l'eau en Camargue}

Du fait de cette situation, la question de savoir ce que peut être un niveau «normal » pour les étangs du système Vaccarès oppose régulièrement les différents acteurs du territoire, notamment ceux qui participent à la commission en charge de la gestion du pertuis. Des représentations diverses s'opposent qui renvoient à la définition que les différents acteurs donnent du territoire de la Camargue. Pour les riziculteurs, la Camargue doit ses paysages et son équilibre aux grands travaux de drainage et d'aménagement qui l'ont vouée à la production agricole, spécialement à la riziculture. La finalité de la Camargue "telle qu’on la connaît» serait la production du riz, dans laquelle l'élevage bovin (taureaux de combat) ou équin n'entre qu'en complémentarité. Pour les naturalistes, la Camargue est un système lagunaire qui, en milieu méditerranéen, doit connaître des périodes d'assèchement, donc des baisses conséquentes du niveau des étangs, ce que ne permettent plus les effluents rizicoles en ayant lieu précisément aux périodes estivales, c'est-à-dire lorsque le système devrait «manquer» d'eau. Les pêcheurs en étang, que certains acteurs gestionnaires considèrent comme "n'ayant qu'une importance patrimoniale" car ne représentant qu'un effectif d'une quinzaine de pêcheurs, forment leurs représentations de ce qu'est le niveau «normal» des

23 Pour une présentation plus détaillée des principes de fonctionnement de la CEDE et des contraintes qu'elle rencontre, on se reportera au texte d'Aurélien Allouche et Laurence Nicolas («Droits liés à l'eau dans la Camargue insulaire: à la croisée de la gouvernance environnementale et de la gestion des risques", Regions \& Cohesion, vol.1, n ${ }^{\circ}$ 3, 2011, p. 67-92), à celui d'Alain Dervieux (op. cit.) et à celui de Raphaël Mathevet et al. ("Water Management in the Camargue Biosphere Reserve: Insights from Comparative Mental Models Analysis", Ecology and Society, vol. 16, n ${ }^{\circ}$ 1, 2011, [en ligne] http://www. ecologyandsociety.org/vol16/iss1/art43/, site consulté en octobre 2014). 
étangs à partir des évolutions de leur activité, en fonction des coins de pêche disparus, de la chute de la ressource halieutique ou encore de la modification du milieu observée au quotidien. Certains acteurs de la protection de la nature ${ }^{24}$ rejoignent la perception de ces pêcheurs en fondant leur jugement sur le transit halieutique d'espèces migratrices dont le «recrutement» dans l'étang est sujet à inquiétude en raison de l'état mondial du stock de ces espèces.

Le travail de la Commission Exécutive de l'Eau consiste donc à tenter de maintenir les étangs du système Vaccarès à un niveau suffisamment bas pour prévenir les risques de débordement et d'inondation ${ }^{25}$, tout en les maintenant suffisamment élevés pour garantir l'exercice de la pêche, la conservation des milieux naturels et la circulation des espèces migratrices, dont l'anguille. On a pu montrer ailleurs que cette situation, en raison des composantes du système technique et de l'absence de critères formels définissant les modalités de satisfaction de ces enjeux, conduisait au travers de processus microsociologiques à une hiérarchisation tacite et informelle des droits des différents usages dépendants de la gestion de l'eau ${ }^{26}$. C'est à un autre point que l'on souhaite s'intéresser ici, mais qui participe également à dessiner tacitement une ligne de devenir du territoire.

La question est en effet de savoir sur quelles connaissances, données et représentations peut être établie la définition d'un état de référence du Vaccarès, et plus spécifiquement d'un niveau d'eau, tenu pour être un état «naturel " à maintenir. En d'autres termes, comment procède l'objectivation de ce territoire dans le cadre de la gestion concertée de ses communications à la mer? Pour les acteurs gestionnaires, la définition de cet état s'entreprend à partir d'une démarche normalisée, standardisée, "objectivée».

24 En particulier, l'association Migrateurs Rhône Méditerranée (MRM) qui siège à la CEDE et dont la principale mission est de veiller à la migration d'espèces telles que l'anguille qui nécessitent d'accomplir une partie de leur cycle de vie à l'intérieur du Vaccarès.

25 Notamment en cas de rupture de digues du Rhône limitrophe dont les eaux doivent pouvoir dans ce cas s'évacuer à la mer à partir du système lagunaire, la situation s'aggravant si le Vaccarès est lui-même à une cote élevée.

26 Aurélien Allouche et Laurence Nicolas, op. cit. 
Plusieurs stations de mesure sont placées à différents points de l'étang pour évaluer son niveau moyen ainsi qu'un appareil de mesure à la sortie du pertuis. Ces données sont mises en rapport avec des moyennes chroniques, croisées avec des suivis de milieu pour les relier à l'état biologique de l'étang. Mais comment les acteurs qui pratiquent cet étang et ses pourtours définissent-ils et évaluent-ils ce que peut être le niveau moyen de l'étang, et plus encore ce que doit être son niveau souhaitable ou "normal»? Comment leurs représentations de ce qu'est ce territoire s'inscrivent-elles dans cet assemblage chronique de moyennes? Quel sens prennent ces relevés de niveau lorsqu'ils sont rapportés, non plus à un plan de gestion d'une réserve naturelle ni à un enjeu écologique, mais à des conceptions de la vie d'un territoire? L'objectivité d'une donnée (telle une moyenne) est-elle une condition suffisante pour assurer le travail d'objectivation du territoire dans le cadre d'une gestion participative?

\section{La dynamique des relations épistémiques entre acteurs dans la reconnaissance, la diffusion et l'utilisation de connaissances}

2.1. Les contours théoriques d'un objet d'étude rendu nécessaire par l'objectivation du territoire

L'objectivation du territoire dans les procédures de gestion participative nécessite une mise en complémentarité des connaissances et des représentations des différents acteurs. Suivant le niveau effectif de participation des individus non décisionnaires (habitants ou usagers) à la mise en œuvre du projet de territoire, l'inscription de leurs connaissances et de leurs représentations dans la définition publique d'un territoire peut être très variable. A minima, c'est-à-dire dans le cas d'une gestion participative se réduisant à une simple consultation (non contraignante pour le décideur) des habitants et des usagers, les représentations et les connaissances de ces derniers ne semblent toutefois pas pouvoir être rigoureusement inopérantes sur la définition du territoire de projet. En effet, elles conditionnent la réception publique du 
projet et le sens qu'il prendra dans l'espace public, tout autant qu'elles peuvent déterminer la viabilité effective de ce projet, lequel peut perdre les fonctionnalités objectives auxquelles il prétendait si ces fonctionnalités ignorent complètement les usages et les représentations en présence.

Si l'on accepte ce qui précède, il semble bien que, même à un niveau élémentaire, la rencontre d'acteurs, experts ou profanes autour de la gestion concertée d'un territoire repose sur une division du travail cognitif d'objectivation du territoire. Une telle division du travail cognitif ${ }^{27}$ est rendue nécessaire par le fait qu'un acteur public peut difficilement réunir et assumer à lui seul l'intégralité des connaissances contextuelles d'un territoire ni l'ensemble des données entrant dans la pratique d'un usager. Ces considérations paraissent d'autant plus pertinentes que la décision collective porte sur une gestion régulière de l'eau qui ellemême obéit à un projet de territoire stipulé par la charte du PNRC. En effet, la dimension spatiale de la gestion rend d'autant plus nécessaire la collaboration cognitive des acteurs que la dépendance mutuelle de chacun d'eux par rapport aux connaissances des autres acteurs s'en trouve accrue.

Or cette division du travail cognitif suppose à son tour un réseau de relations épistémiques entre les acteurs, relations qui se résument à l'autorité, la dépendance et la confiance épistémiques. Ces concepts ont été développés (par l'épistémologie sociale) principalement pour penser le recours à une connaissance par témoignages (testimonies) et la fiabilité de connaissances auxquelles on ne peut accéder que par l'entremise d'un expert. Ces facteurs épistémiques forment en eux-mêmes un ensemble causal irréductible aux autres paramètres sociaux, ce qui leur vaut une attention croissante de la part de la sociologie, même si les travaux de cette espèce observent une diffusion encore quelque peu restreinte ${ }^{28}$.

\section{$27 \quad$ Philip Kitcher, op. cit.}

28 Les relations épistémiques ont été explicitement mobilisées dans divers travaux de sociologie, par exemple par Emmanuel Lazega ( Pertinence et structure», Swiss Jounal of Sociology, vol. 37, $\mathrm{n}^{\circ}$ 1, 2011, p. 127-149) pour penser une sociologie néo-structuraliste ou pour décrire des pratiques profes- 
La confiance épistémique, c'est-à-dire la confiance que l'on place dans une donnée ou dans la source qui l'émet, a pu être abordée initialement en sociologie sans recourir explicitement à cette notion, l'inscrivant plus généralement dans une réflexion sur la confiance comme ressource permettant de gérer collectivement des flux d'information ou des dispositifs techniques sans avoir besoin de les contrôler ou de les connaittre intégralement. Il est alors question de la confiance comme mécanisme de réduction de la complexité sociale ${ }^{29}$ ou encore de la confiance ontologique $^{30}$ désignant par-là l'absence de doute et de questionnement quant au bon fonctionnement des systèmes experts.

John Hardwig a très largement initié un courant de réflexion portant sur la dépendance épistémique et sur les autorités épistémiques. Il part du constat que pour nombre de nos croyances nous ne sommes pas en situation de pouvoir en livrer une preuve indiscutable, ni même de pouvoir les mettre à l'épreuve systématiquement. Pour autant, ces croyances ne nous paraissent pas relever de la simple opinion. Cette situation étant très courante, Hardwig s'est demandé s'il pouvait y avoir de bonnes raisons de croire en des propositions pour lesquelles nous n'avons ni preuve

sionnelles particulières telles que l'entretien médical (Gloria Origgi et Marta Spranzi «La construction de la confiance dans l'entretien médical», dans Thierry Martin et Pierre-Yves Quiviger (dir.), Action médicale et confiance, Besançon, Presses universitaires de Franche-Comté, 2007, p. 227-246). Dans l'étude de l'espace public et de la démocratie délibérative, les relations épistémiques fournissent un objet de questionnement de longue date. Ainsi James Bohman rappelle que John Dewey pointait du doigt les conséquences sur la démocratie de l'«interdépendance épistémique» née de la division du travail, alors qu'il considère lui-même les démocraties contemporaines comme spécifiquement marquées par un "pluralisme épistémique» ("Réaliser la démocratie délibérative comme mode d'enquête: le pragmatisme, les faits sociaux et la théorie normative", Tracés, vol. 15, no 2, 2008, p. 1-29). Dans le cadre d'étude de la démocratie "participative» ou "délibérative», des analyses des relations épistémiques entre acteurs ont apporté des résultats notables, notamment pour l'étude de situations de débat public, de controverses scientifiques et plus largement en épistémologie sociale (Alban Bouvier et Bernard Conein (dir.), L'épistémologie sociale : une théorie sociale de la connaissance, Éditions EHESS, Paris, 2007).

29 Niklas Luhmann, La confiance. Un mécanisme de réduction de la complexité sociale, Paris, Economica, 2006.

30 Anthony Giddens, op. cit. 
ni possibilité d'en trouver par nous-mêmes. L'auteur aboutit au résultat a priori paradoxal qu'il peut être parfaitement rationnel, sous certaines conditions, de suspendre son sens critique et d'accepter ces propositions. Il est parfois irrationnel de vouloir penser par soi-même ${ }^{31}$. Résultat quelque peu provocateur dans sa forme, mais qui permet de comprendre la généralité d'un comportement mental, tellement courant, et de ne pas le traiter comme anormal ou irrationnel. Nous croyons nombre de propositions que nous ne sommes ni en état d'investiguer ni en capacité d'établir par une preuve, parce que nous avons de bonnes raisons de croire qu'une autorité épistémique donnée (tel expert, telle personne, etc.) a elle-même de bonnes raisons de croire ces propositions et qu'elle a conduit les enquêtes nécessaires pour cela.

La relation entretenue à une autorité épistémique, c'est-à-dire la nécessité dans laquelle on est de devoir en appeler à elle pour justifier ou évaluer une croyance ou une connaissance, est caractéristique d'une dépendance épistémique. De plus, il ne nous suffit pas de croire dans le statut d'autorité épistémique d'un acteur donné pour en faire la justification à l'acceptation d'une proposition. Il faut également croire qu'il exerce effectivement bien son rôle, et non pas simplement qu'il en est capable. En somme, la confiance épistémique doit accompagner la reconnaissance du statut d'autorité épistémique. John Hardwig accentue le rôle de la confiance épistémique dans la connaissance en faisant de la possibilité de faire confiance aux membres d'une communauté épistémique la fondation dernière de la plupart des connaissances.

Or, de ce point de vue, les notions d'autorité, de confiance et de dépendance épistémiques peuvent légitimement être employées à une échelle microsociologique, sans se cantonner pour autant aux débats opposant connaissances expertes et profanes et sans non plus identifier les compétences épistémiques aux seuls savoirs

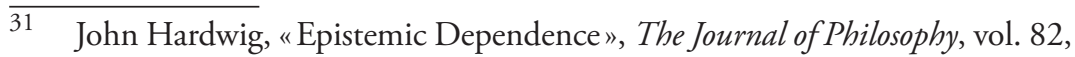
$\mathrm{n}^{\circ}$ 7, 1985, p. 335-349; John Hardwig, "The Role of Trust in Knowledge», The Journal of Philosophy, vol. 88, $\mathrm{n}^{\circ}$ 12, 1991, p. 693-708. 
scientifiques. Autorité, confiance et dépendance épistémiques forment un triptyque particulièrement pertinent pour une analyse microsociologique. La dépendance épistémique définit un état situationnel, lié en particulier à une division du travail cognitif ${ }^{32}$ ou une division épistémique du travail, dans lequel des acteurs s'inscrivent. L'autorité et la confiance ${ }^{33}$ épistémiques relient ces acteurs, mais ne sont pas des données immuables ou intangibles, elles sont fortement liées à un mode de présentation de soi et dépendent d'une évaluation rationnelle et objectivante des occurrences de manifestation des compétences cognitives et épistémiques des acteurs prétendant à l'autorité épistémique.

Certes, les acteurs sont, du fait de la division du travail cognitif, dans une situation de dépendance envers des autorités épistémiques, mais ils n'adoptent pas de manière automatique les jugements de celles-ci et y accordent un degré variable de confiance. Si un individu ne peut pas directement constituer un jugement sur la valeur des propositions communiquées par une autorité épistémique, il pourra établir un jugement sur le contexte dans lequel s'exprime cette autorité ou encore sur les qualités de celle-ci (son honnêteté intellectuelle, par exemple). De ce fait, la dynamique des relations épistémiques est un objet essentiel pour comprendre la formation de consensus et l'évolution des désaccords dans un collectif débattant d'un problème public, comme Alban Bouvier ${ }^{34}$ a pu le montrer en prenant pour

32 Philip Kitcher, op. cit.

33 La relation de confiance lorsqu'elle est non épistémique est déjà en ellemême, selon Georg Simmel (Secret et sociétés secrètes, Belval, Circé, coll. «Poche», 1991 [1906]) un état intermédiaire entre savoir et non-savoir qui en fait une "des forces de synthèse les plus importantes au sein de la société» puisque celui qui sait tout d'un être humain n'a pas besoin de faire confiance et celui qui ne sait rien ne peut pas même faire confiance. S’il ne s'agit pas pour nous de glisser ici d'une acception de la confiance à celle de la confiance épistémique, il est à noter toutefois que les propriétés intrinsèques de la confiance supposent chez l'acteur une synthèse réflexive entre ce qu'il sait et ce qu'il ne sait pas, qui le rend tout à la fois dépendant et indépendant de la personne à qui il choisit d'accorder sa confiance. En ce sens, il n'est pas irréaliste d'y voir une relation, plutôt qu'un état.

Alban Bouvier, "La dynamique des relations de confiance et d'autorité au sein de la démocratie dite "participative" et "délibérative" ", Revue européenne 
objet le débat public relatif à l'installation d'une ligne électrique à très haute tension dans la région Provence-Alpes-Côte-d'Azur ${ }^{35}$.

L'hétérogénéité des connaissances et des observations discutées dans le cadre de la gestion participative d'un territoire, du fait de la diversité des domaines de savoir qui y sont mobilisés et du degré d'expertise des acteurs, suggère que la dynamique des relations épistémiques est cruciale dans l'articulation des connaissances des usagers et des habitants avec celles des acteurs gestionnaires ou experts.

2.2. La dynamique des relations épistémiques dans la gestion participative de l'eau en Camargue

L'observation de trois années de fonctionnement de la Commission Exécutive de l'Eau montre l'existence de liens essentiels entre la dynamique des relations épistémiques et le mode de socialisation des connaissances au sein de la gestion participative. Ces liens nous ont paru conditionner la participation de certains usagers. Il est possible de synthétiser ces observations à partir de l'analyse d'une situation s'étant déroulée en séance (réunion du 15 juillet 2009), où une opposition s'est élevée entre un pêcheur et un acteur gestionnaire, donnant lieu aux échanges retranscrits cidessous (voir l'encadré 1).

Dans des échanges précédant la partie retranscrite, ce gestionnaire exposait les relevés de niveau des étangs du système Vaccarès et affirmait que celui-ci était à un niveau élevé par rapport à son niveau moyen. Il incitait les différents acteurs à préconiser l'ouverture du pertuis pour abaisser le niveau de l'étang afin d'anti-

des sciences sociales, vol. 45, $\mathrm{n}^{\circ}$ 136, 2007, p. 181-230; Alban Bouvier, «Dimensions axiologique, épistémologique et cognitive de la délibération publique : analyse d'un exemple ", Cahiers d'économie politique, n ${ }^{\circ} 47,2004$, p. $215-234$.

35 Nous renvoyons également à Allouche ( $«$ Les relations épistémiques de confiance et d'autorité dans l'action collective et le militantisme. Exemple par la sociologie des conflits environnementaux", Implications Philosophiques, numéro spécial, «Modernité et confiance», 2014 [en ligne] http:// www. implications-philosophiques.org, consulté en octobre 2014) pour une application de cette réflexion à un autre cadre empirique, celui d'un conflit d’aménagement dans la région de l'étang de Berre (Bouches-du-Rhône, France). 
ciper de possibles pluies importantes qui ne manqueraient pas d'arriver et qui compromettraient les possibilités de maintenir des cotes acceptables au regard du risque de débordement de l'étang. Le pêcheur, qui prend la parole dans l'extrait retranscrit, s'inquiète de cette préconisation, d'où sa demande (au tour de parole 1) de ne pas évacuer trop d'eau à la mer par fort vent du nord. Ce pêcheur, qui exerce son activité dans la partie sud des étangs inférieurs, sur la commune des Saintes-Maries-de-laMer, considère pour sa part que le niveau de l'étang, s'il est supérieur aux moyennes annuelles et interannuelles, n'en est pas moins anormalement bas, des zones demeurent asséchées alors qu'elles ne devraient pas l'être.

Cet acteur est en situation de devoir formuler une préconisation de gestion à partir de données issues de stations de mesure, données qui sont en contradiction avec l'observation quotidienne qu'il fait de l'étang au cours de son activité professionnelle. De surcroît, il connaît mal les protocoles de mesure mis en place par le gestionnaire, ainsi que les procédés d'extrapolation du niveau moyen de l'étang à partir d'un quadrillage de stations de mesure, disséminées sur l'étang. L'enjeu de l'échange analysé consiste à rétablir la confiance dans des données et l'autorité épistémique d'un acteur lorsqu'une dissonance est perçue entre ces données et l'expérience qu'un usager a de son territoire. Or l'objectivation du territoire suppose de tenir pour acquis, pour "évident», un certain nombre de données partagées. Que se passe-t-il alors?

Encadré 1:

Extrait de la réunion de la CEDE du 15 juillet 2009

1. PECHEUR: Il faut éviter de faire sortir par coup de mistral maintenant. Parce que maintenant y a qu'à attendre

2. PRESIDENT DE SEANCE: on attend?

3. PECHEUR: Ben, il faut bien on a sorti tellement d'eau que maintenant, faudrait qu'il va pleuvoir

4. PRESIDENT DE SEANCE: on est -on est haut quand même

5. PECHEUR: $<$ Rire $>$ me dites pas ça, c'est pas possible $<$ Rire $>$

6. PRESIDENT DE SEANCE: attendez, si si - 
7. PECHEUR: on n'est pas à un niveau haut $<$ Rire>

8. PRESIDENT DE SEANCE: on est à un niveau largement plus haut que la moyenne

9. ACTEUR GESTIONNAIRE: moi, je sais pas à quoi ça sert d'envoyer des papelards et de faire des mesures. Moi, je rends mon tablier et puis voilà!

10. PRESIDENT DE SEANCE: [avec empressement] non, mais je pense qu'il faut redire effectivement qu'on est à un niveau -

11. ACTEUR GESTIONNAIRE: si c'est pour entendre - moi je rends mon tablier, vous vous démerdez et puis vous leur demandez à eux les mesures

12. ACTEUR GESTIONNAIRE: si c'est ça!

13. PECHEUR: ah! ben non, mais -

14. ACTEUR GESTIONNAIRE: si c'est ça, j'étais ce matin en train de mesurer -

15. PECHEUR: ben, si vous voulez, moi, je vous amène, vous verrez l'étang qu'il y a maintenant, vous verrez

[l'acteur gestionnaire se lève et quitte la réunion]

16. PECHEUR: y a des baisses ${ }^{36}$ où y a plus d'eau du tout. Moi je le vois, l'étang j'y suis tous les jours, alors c'est vrai que peut-être pour ses mesures - mais, moi, je parle pas en mesures, moi quand le niveau 0 , c'est le niveau de la mer. Quand il me dit, il est $14 \mathrm{~cm}$ au-dessus du niveau de l'étang c'est pas normal! attendez!

17. PRESIDENT DE SEANCE: laissez-moi terminer, s'il vous plait. Donc ce que vous venez dire effectivement est faux <accentue le mot «faux" >. On est largement au-dessus des moyennes habituelles. On est largement au-dessus - même on est au-dessus de la plage - on est dans la plage accidentelle de variations euh qui est prévue par le plan de gestion au niveau du Vaccarès. Donc ce qui est mesuré est mesuré. On va pas le contester. Après on peut se poser des questions en termes d'évolution. Alors après ce qui se passera au 15 septembre je ne sais pas, on le constatera. Ce qui me parait clair, ce qui est non contestable, c'est qu'effectivement les niveaux sont beaucoup plus hauts que d'habitude. On part d'une situation où effectivement les niveaux étaient hauts, on a fait des efforts pour sortir de l'eau. On a sorti de l'eau et effectivement les salinités ont baissé, mais les niveaux restent hauts

18. PECHEUR: non, ce que je veux pas dire, c'est pas que le niveau est plus haut que l'année dernière. Ce que je veux dire c'est que ça fait deux ans qu'on était en sécheresse! Donc les niveaux qui sont pas -

19. PRESIDENT DE SEANCE: non je parle pas - non je parle pas de l'an dernier, je parle de moyennes

36 Dépressions topographiques qui s'emplissent d'eau. 
En somme, cet échange est caractéristique d'une situation de dépendance épistémique, dans la mesure où le pêcheur doit justifier ses préconisations sur la base de données dont la commission exige de lui qu'il les tienne pour vraies. Notons d'abord que la situation de dépendance épistémique dans laquelle se trouve ce pêcheur est partagée par la majorité des membres de la CEDE. La plupart d'entre eux acceptent l'agrégation des données produites par le gestionnaire comme valant pour le niveau moyen de l'étang, sans connaître ces modalités d'agrégation, ni les conditions dans lesquelles les relevés de ces mesures sont réalisés. Les membres s'en remettent à ces mesures et à leurs producteurs en substituant aux raisons de faire confiance aux données celles de faire confiance à leurs producteurs et au contexte institutionnel où s'insèrent la récolte et l'utilisation des mesures ${ }^{37}$.

Pour autant, les propos de ce pêcheur ne sont pas un refus du travail d'objectivation puisqu'il donne au contraire des éléments objectivables et ne se limite pas à un argument d'autorité ni à la revendication du caractère irréductible de son expérience personnelle. Il mentionne, par exemple, l'existence de "baisses", connues pour être à cette saison en eau et qui sont alors asséchées. Il se dit prêt à montrer sur le terrain les éléments qui lui font dire que l'étang ne peut pas être tenu pour «haut». Sa démarche pourrait s'inscrire dans un travail collectif d'objectivation auquel

37 On peut faire ici une mise en continuité avec la sociologie de la traduction, dans la mesure où l'utilisation des données produites par le réseau de mesures pour élaborer et justifier des préconisations de gestion apparaît comme un point de passage obligé. Celui-ci résulte de la collaboration de nombreux acteurs, mêlant aussi bien les techniciens d'une réserve naturelle que les scientifiques d'une fondation publique de conservation et de recherche, laquelle possède certaines des stations de mesure et inclut l'utilisation de ces mesures dans des programmes de recherche subventionnés. De même, les décisions de gestion s'établissent en considérant le déplacement des flux halieutiques entrant dans la lagune ou en sortant, déplacement lui-même conditionné par les tentatives des différents modes de gestion de l'orienter vers les endroits qui usuellement servent à en mesurer l'importance, que ce soit par des engins de pêche ou par des stations de mesure. Néanmoins, nous ne nous intéressons ici davantage à la gestion par les acteurs de la contextualisation de ces dispositifs dans le temps spécifique de la concertation. 
participeraient alors ses représentations d'un état «normal» de la lagune. Pourtant la Commission ne va pas entamer ce travail.

Cela s'explique parce que c'est un autre enjeu qui se joue dans ces échanges, et ce, sous une forme conflictuelle, notamment avec la sortie de l'acteur gestionnaire qui affirma dans les jours qui suivirent ne plus vouloir «remettre les pieds» dans la Commission tant que ce pêcheur y sera (la situation se pacifiera par la suite). Ces échanges procèdent d'une régulation de l'autorité épistémique et montrent clairement les relations micro-interactionnelles d'établissement de cette autorité épistémique. En effet, les interventions du pêcheur sont closes par le président de séance qui ferme l'échange sur l'affirmation du caractère incontestable des mesures. Le président de séance invalide (les autres membres ratifient tacitement cette invalidation) le jugement du pêcheur : "ce que vous venez dire, effectivement, est faux" (tour de parole 17). Lautorité épistémique est refusée au pêcheur, auquel il n'est donné aucune réponse aux éléments factuels qu'il énonce tel que l'assèchement des baisses.

L'empressement du président de séance à dépasser le conflit en invalidant le jugement du pêcheur est à comprendre au regard de la contrainte de confiance épistémique qui prévaut au travail collectif d'objectivation. L'enjeu est de préserver une source de données à laquelle les acteurs peuvent s'en remettre, même si certains d'entre eux ignorent partiellement le mode de production de ces mesures. Il s'agit de préserver des connaissances d'arrière-plan, des données partagées qui n'ont plus besoin d'être discutées et peuvent au contraire rendre possibles la discussion et la décision collective. C'est en ce sens que le président de séance insiste: "ce qui est mesuré est mesuré». La reconnaissance à un acteur (le gestionnaire) d'une autorité épistémique permet corrélativement d'établir un rapport de confiance envers les jugements ou les données qui émanent de lui. Cette confiance épistémique est à son tour indispensable aux autres acteurs pour leur permettre une réflexivité sur ce qu'ils savent ignorer mais qu'ils peuvent néanmoins choisir d'accepter en se remettant à cette autorité et aux critères qui l'attestent. 
Cette logique, rationnellement fondée ${ }^{38}$, tend à rendre la gestion collective du territoire plus opérante grâce à une division du travail cognitif. Toutefois lorsqu'elle infléchit non seulement la dimension cognitive des échanges, mais aussi leur dimension interactionnelle, on peut se demander si les dynamiques d'autorité épistémique ne tendent pas à ralentir la constitution de connaissances partagées (d'arrière-plan). Une observation nous incite à en faire l'hypothèse.

En effet, en mai 2012, une réunion de la Commission de gestion de l'eau et de la CEDE est organisée au PNRC. Y sont présentés les résultats d'une étude sur les anguilles, réalisée par Alain Crivelli, chercheur à la fondation de la Tour-du-Valat, lequel attire tout particulièrement l'attention des différents membres de la CEDE sur deux points essentiels. Certes, l'ouverture maximale du pertuis de la Fourcade (ouverture de douze ou treize vannes) permet un important "appel en mer" pour «recruter» des civelles ${ }^{39}$, en créant un panache d'eau douce s'étendant loin en mer que les civelles vont suivre jusqu'à entrer dans les étangs. Cela parait a priori favorable aux intérêts des pêcheurs autant qu'aux objectifs des naturalistes. De plus, cette ouverture maximale correspond aux modalités de gestion appliquées par la CEDE lorsqu'elle décide de maximiser les rejets en mer en situation de fort vent de secteur nord-nord-ouest (mistral), modalités que certains pêcheurs critiquent. Cette observation scientifique semble rendre caduque la demande des pêcheurs de ne pas abaisser trop fortement ni trop rapidement le niveau des étangs centraux en ouvrant complètement le pertuis les jours de fort mistral. Certes, l'ouverture importante diminue le niveau d'eau des étangs, mais elle permet, en peu de temps, de «recruter» massivement des civelles.

Or Alain Crivelli met en garde. D'une part, le débit occasionné par l'ouverture totale du pertuis s'avère trop fort pour que les civelles puissent le remonter. Elles sont "appelées ", mais en vain. D'autre part, l'ouverture maximale du pertuis est souvent suivie

\footnotetext{
38 John Hardwing, "The Role of Trust in Knowledge», op. cit.

39 Les civelles sont les alevins d'anguilles.
} 
de sa fermeture complète : les vannes sont ouvertes durant une courte période, lorsque le vent est fort, puis le pertuis est refermé pour ne pas perdre l'abaissement du niveau des étangs gagné, puisque, le mistral cessant, le niveau de la mer redevient supérieur à celui des étangs. Les civelles appelées se retrouvent alors devant une porte fermée. Le recrutement ne se fait pas ou se fait mal.

Ces deux informations, présentées lors d'une réunion initialement pensée pour transmettre de l'information aux acteurs dits "de la société civile», interpellent surtout deux membres gestionnaires de la CEDE, le président de la commission de gestion de l'eau et le directeur adjoint du PNRC. Ces personnes en tirent immédiatement les conséquences : la gestion des vannes devra changer sur ces deux points. Un pêcheur soupire : il aura fallu tout ce dispositif scientifique pour arriver à ce qu'il savait déjà. Et en effet, près de deux ans auparavant, celui-ci nous expliquait en entretien :

Donc, le problème, c'est que soit vous avez trop d'eau qui sort d'un coup, ça a trop de courant. D'ailleurs, il l'a dit le jeune de MRM, il a dit sil y a trop de courant, le poisson il ne rentre pas. Donc en fait, comme on fait énormément sortir quand il y a des coups de mistral, c'est avantageux parce qu'on va faire baisser le niveau au maximum. Eh ben, ça attire énormément le poisson. Ce quill faudrait après, c’est laisser ouvertes deux-trois matillières ${ }^{40}$ pour que ce poisson, qui est attiré, puisse rentrer.

Deux éléments apparaissent. On peut voir que le pêcheur interviewé fait référence au travail communiqué par l'association "Migrateurs Rhône Méditerranée» (MRM) lors de plusieurs réunions de la CEDE. De ce point de vue, l'opérationnalisation de cette connaissance dans la gestion et son appropriation par des acteurs gestionnaires auront nécessité une présentation, bien plus tard, de ces mêmes résultats par un scientifique reconnu. Il semblerait que le pêcheur interviewé n'a pas pu trouver l'occasion lui permettant de communiquer clairement cette information. Ses évocations de cette donnée au cours des réunions de la CEDE n’ont pas été relevées par les autres acteurs. En effet, le principal mode d'interpellation des acteurs au sein de la CEDE demeure $\overline{40}$ Terme local désignant les vannes. 
la formulation de préconisations de gestion, or le mode d'expression privilégié par ce pêcheur y est davantage celui d'une prise de position, voire d'une opposition, car il sait sa position être minoritaire. Il communique avec empressement, n'est parfois pas écouté ou encore se résigne et renonce à insister pour faire entendre sa demande. Plus exactement, ses prises de parole sont reçues sur le mode d'une demande ou de la défense d'une position, en raison, notamment, de son usage de phrases "choc" (fortes marques énonciatives, hyperboles, valeur perlocutoire marquée, prosodie explicite, etc.) telles que : «bientôt, je pourrai aller pêcher en baskets tellement les niveaux sont bas!». Ses interventions, surtout au début de sa "carrière» de membre, sortent parfois du cadre des conventions tacites qui régissent la prise de parole en réunion, reprenant, par exemple, la parole alors que le président de séance tente de la faire circuler. L'ensemble de ces éléments cadre peu avec les échanges du type de ceux orientés par le partage des connaissances. De fait, ce pêcheur ne parvient pas à se faire reconnaître une autorité épistémique en matière de peuplement piscicole, ce qui pèse sur le mode d'interaction entretenu par le groupe avec lui. Il en résulte dans cet exemple que la CEDE mettra deux ans avant d'accéder à une connaissance pourtant présente parmi ses membres, mais encore non disponible en tant que connaissance d'arrière-plan, car n'ayant pas rencontré un processus d'autorisation épistémique.

De ce point de vue, les relations épistémiques entre acteurs semblent importantes dans la capacité du territoire objectivé à permettre une rencontre entre le territoire de projet et le territoire vécu. L'objectivation du territoire n'est donc en aucun cas à rabattre du côté d'une catégorie d'acteurs au détriment d'une autre. Elle renvoie davantage à une attitude épistémique à travers laquelle des acteurs tentent de donner des indices et des référents transsubjectifs qui permettent à leur tour d'équilibrer les rapports d'autorité et de confiance épistémiques. Une observation reproductible, falsifiable, standardisée, "objective» peut parfaitement échouer à s'inscrire dans un processus d'objectivation du territoire si elle ne permet pas à l'ensemble des acteurs d'émettre un 
jugement sur l'espace délimité, approprié, pratiqué et socialisé. Si cette même observation, aussi objective soit-elle, sert des relations d'autorité épistémique fortement déséquilibrées, elle peut paradoxalement desservir l'objectivation collective du territoire.

\section{Les critères qui fondent les représentations issues du territoire et leur inscription dans un cadre objectivé}

Les dynamiques des relations épistémiques au sein de la gestion participative du territoire ne recouvrent pas l'ensemble des phénomènes jouant dans la capacité des usagers et des habitants à inscrire leurs représentations et leurs connaissances dans le processus général d'objectivation du territoire. Si des facteurs interactionnels (micro-interactionnels) interviennent, un second type d'explication doit également être mis en exergue. Les conditions d'expression et d'organisation de ces connaissances et de ces représentations doivent être interrogées. Revenons pour cela sur l'échange précédent (voir l'encadré 1).

Le président de séance et les autres membres de la commission, une fois établi le fait que les mesures du niveau de l'étang ne sont pas à remettre en question, ne cherchent pas à éclairer les éléments d'incompréhension qui ont conduit à l'altercation entre le gestionnaire et le pêcheur. Ils ne semblent pas non plus vouloir faire expliciter la représentation divergente de ce dernier.

Comment interpréter en effet cette incompréhension mutuelle qui n'est pas sans conséquence sur les possibilités de constitution de connaissances d'arrière-plan partagées? Le président de séance semble l'expliquer en considérant que le pêcheur n'a pas à sa disposition la notion de moyenne, insistant notablement sur ce point (tour de parole 19). Un entretien avec ce pêcheur et des conversations informelles montrent que cette interprétation est erronée. Mais alors, comment expliquer cette incompréhension mutuelle?

Le pêcheur ne ratifie en fait pas l'usage de la moyenne comme cadre pertinent pour évaluer ce que doit être le niveau du Vaccarès. La différence est de taille, car, dans l'échange, persuadé 
d'un défaut conceptuel du pêcheur, le président de séance coupe court sur cette notion, en conséquence de quoi le cadre de pertinence du pêcheur n'est absolument pas approfondi par les autres acteurs. Cet état de fait s'explique également par l'enjeu organisationnel de maintenir la reconnaissance de la légitimité de l'acteur gestionnaire tout autant que de conserver des données chiffrables et reconnues de tous pour fonder la décision.

Si le pêcheur n'emploie pas la notion de niveau moyen, c'est qu'il lui préfère celle de niveau «normal». Qu'entendre par cette normalité? Sur quels savoirs s'établit l'estimation de ce qu'est une cote normale? Et comment la connaissance et les représentations de ce pêcheur peuvent-elles s'intégrer au processus d'objectivation du territoire?

Le niveau «normal» pour le pêcheur est celui pour lequel la fonctionnalité du système est assurée. Lors d'un entretien, il nous montre un pertuis complètement enlisé et rendu hors d'usage, marquant pour la seule mémoire une connexion hydraulique qui ne se fait plus. Il nous parle de ces baisses qui ne se remplissent plus, et qui le devraient "normalement", sans quoi, comment expliquer leur existence? Le niveau normal n'est alors pas défini à partir de séries chroniques de mesures dont pourrait être extraite une moyenne interannuelle, auquel cas le niveau «normal» serait le niveau «régulier» ou "courant», et pêcheurs et gestionnaires naturalistes partageraient de facto la même référence. Pour ce pêcheur, le niveau normal est celui pour lequel l'étang parvient à fonctionner. C'est le niveau qui rend possibles les échanges hydrauliques et halieutiques entre l'étang et la mer, échanges qui sont tenus pour nécessaires à la définition d'une lagune. Le «normal» ne se comprend pas, dans ce cadre, comme l'antonyme de l'«accidentel» ou de l'«exceptionnel », ainsi que ce pourrait être le cas dans une définition en termes de «moyenne». L'antonyme est bien celui d' "anormal» ou de "dysfonctionnel».

Le pêcheur définit également le niveau normal à partir d'une complémentarité fonctionnelle des sous-ensembles territoriaux. Il explique en effet durant l'entretien : 
C'est-à-dire que même maintenant avec des niveaux qu'on avait, qui étaient pas très hauts, on devait faire [sortir de l'eau] parce qu'il fallait qu'on soit à -10 , on était à ce moment-là à 0 ou 10 , je me rappelle plus. Si chaque fois dès qu'il pleut on veut faire sortir, on n'arrivera jamais à avoir un système qui se faisait comme avant. Avant, on recevait énormément d'eau des rizières, donc quand on arrivait en septembre, on avait énormément d'eau. On savait pas d'où elle sortait, généralement ça tombe en avril. Donc tout l'hiver, on pêchait avec un bon niveau d'eau, et au printemps l'eau était suffisamment haute pour attirer le poisson et on repartait comme ça. C'est vrai que des fois on avait des étés qui étaient un petit peu justes, mais l'hiver, nous on avait un niveau d'eau qui se faisait, donc on n'avait pas de problème. Pendant des années, on a fait comme ça.

On saisit dans cette citation que la perception du niveau «normal» des étangs condense une représentation de la complémentarité des usages, évoquée par l'apport des eaux rizicoles qui permettent à l'ensemble de fonctionner, ajoutant en cela une dimension sociale aux fonctionnalités écologiques du système. Plus loin dans l'entretien, c'est aussi le gibier d'eau qui est évoqué, les canards et autres bêtes à plumes qui ne se chassent plus tellement à l'extrême sud, alors qu'avant il s'en chassait tant. C'est donc que quelque chose ne fonctionne pas, qu'on ne garde pas assez longtemps l'eau.

En somme, la représentation portée par un pêcheur de ce que devrait être le niveau de l'étang s'appuie sur une conception complexe et holistique du territoire et du système hydraulique qui aborde solidairement les activités humaines, la complémentarité des sous-systèmes territoriaux et les dynamiques écologiques. La complexité d'une telle conception, sinon son syncrétisme, peut difficilement s'exprimer dans le temps d'une réunion, aux objectifs très pratiques et aux modalités de décision routinisées. À défaut de cela, les autres acteurs (gestionnaires ou non) peuvent vouloir disqualifier cette conception au cours de l'objectivation collective du territoire, en lui opposant des moyennes de mesures bathymétriques ou piézométriques, plus 
facilement communicables et immédiatement compréhensibles ${ }^{41}$ dans le cadre des échanges de la CEDE.

Paradoxalement, cette conception du "normal ", indépendamment de la question de son caractère erroné ou non, pourrait offrir au travail collectif d'objectivation des opportunités bien plus importantes que ce que le président de séance semble le supposer dans l'extrait de réunion analysé. La perception "profane» du niveau normal de l'étang présente ici des points de convergence avec, par exemple, une approche de la conservation par l'écologie fonctionnelle ${ }^{42}$, laquelle représente un tournant paradigmatique en privilégiant la préservation des fonctions écologiques par rapport à la défense d'états naturels invariables et d'espèces emblématiques. Il y a donc en cela un "creuset» d'intersubjectivité appréciable pour la convergence des acteurs dans la définition de ce qui peut être tenu pour un niveau acceptable de l'étang du Vaccarès ${ }^{43}$.

Dans l'extrait de réunion analysé, le président de séance et les autres participants ne se mettent pas en situation de percevoir un possible rapprochement entre cette conception profane du fonctionnement écologique et hydraulique du système lagunaire et sa conception scientifique et technique. Cela semble s'expliquer plus largement par le présupposé ${ }^{44}$ des membres de la CEDE d'une séparation rigide entre savoirs profanes et savoirs experts qui

41 Dans une lecture par la sociologie de la traduction, on pourrait peut-être interpréter cette situation comme relevant de la capacité des acteurs qui appuient leur position sur un dispositif de traduction à intégrer la position d'un usager qui ne mobilise aucun dispositif et refuse les points de passage obligés.

42 Peter Calow, "Towards a Definition of Functional Ecology», Functional Ecology, vol. 1, n 1, 1987, p. 57-61.

43 Précisons bien que notre propos ne consiste pas à tenir les différentes représentations et connaissances comme étant toutes de même nature, équivalentes ou correctement fondées. Il s'agit simplement de signifier que le fait de réunir les conditions d'une expression plus complète de connaissances vernaculaires permet de faire apparaître des points de convergence entre acteurs, points à partir desquels, éventuellement, des représentations contestées pourront être révisées.

Plus qu'un présupposé, il s'agirait d'une conjecture implicite au sens de Boudon (L'art de se persuader des idées douteuses, fragiles ou fausses, Paris, Fayard, 1990). 
n'engage pas les acteurs à approfondir le point de vue défendu par le pêcheur.

Le dépassement de cette situation par les membres de la CEDE ne serait possible qu'à condition que des éléments objectivables, à l'image des relevés de mesure, soient présentés à l'appui des dires du pêcheur. Des nouveaux rapports de confiance et d'autorité épistémiques pourraient alors être construits sur la base de tels éléments. En effet, les connaissances profanes ne visent pas une formalisation systématique ${ }^{45}$ et reposent sur des éléments issus de l'observation directe et quotidienne. De ce fait, elles sont régulièrement perçues par les acteurs experts comme entachées de subjectivité, par opposition à la reproductibilité d'observations accomplies dans le cadre de protocoles de mesure. Or, les éléments les plus objectifs ne sont pas nécessairement les éléments observables par le plus grand nombre d'acteurs. La gestion participative peut néanmoins nécessiter ces deux dimensions (l'objectif et l'observable) si elle souhaite impliquer la plus grande diversité d'acteurs. Le risque serait donc grand pour elle de se confronter à une dualité stricte entre des connaissances profanes, directement observables, mais irréductiblement subjectives, et des connaissances expertes, objectives mais nécessitant la médiation de dispositifs scientifiques et techniques pour y accéder.

Pourtant, si l'évaluation profane du niveau «normal» recourt à l'observation de la pratique et à la mémoire sociale, elle n'est pas non plus sans employer des repères objectifs, ou "objectivables", c'est-à-dire que chacun peut voir dans les mêmes conditions, même si les interprétations du sens à leur donner peuvent diverger.

L'un de ces repères est immédiatement issu de la pratique professionnelle, en prise avec la matérialité du territoire. Les pêcheurs du Vaccarès utilisent des piquets de bois, soit pour fixer leurs filets, soit pour matérialiser des emplacements dans l'étang qui leur sont réservés. Ils peuvent ainsi accéder à un repère fixe dont il est peu douteux qu'il matérialise la présence ancienne d'eau. L'interprétation qu'en donne le pêcheur (suivi sur son lieu

45 Jean-Blaise Grize, op. cit. 
de travail) est sans équivoque, ces piquets étaient, il y a quelques années, dans l'étang et sont aujourd'hui entourés de végétation (voir les figures 2 et 2 bis).

Figure 2 et 2 bis :

Vue des piquets vers l'intérieur des terres; vue sur l'étang depuis les piquets
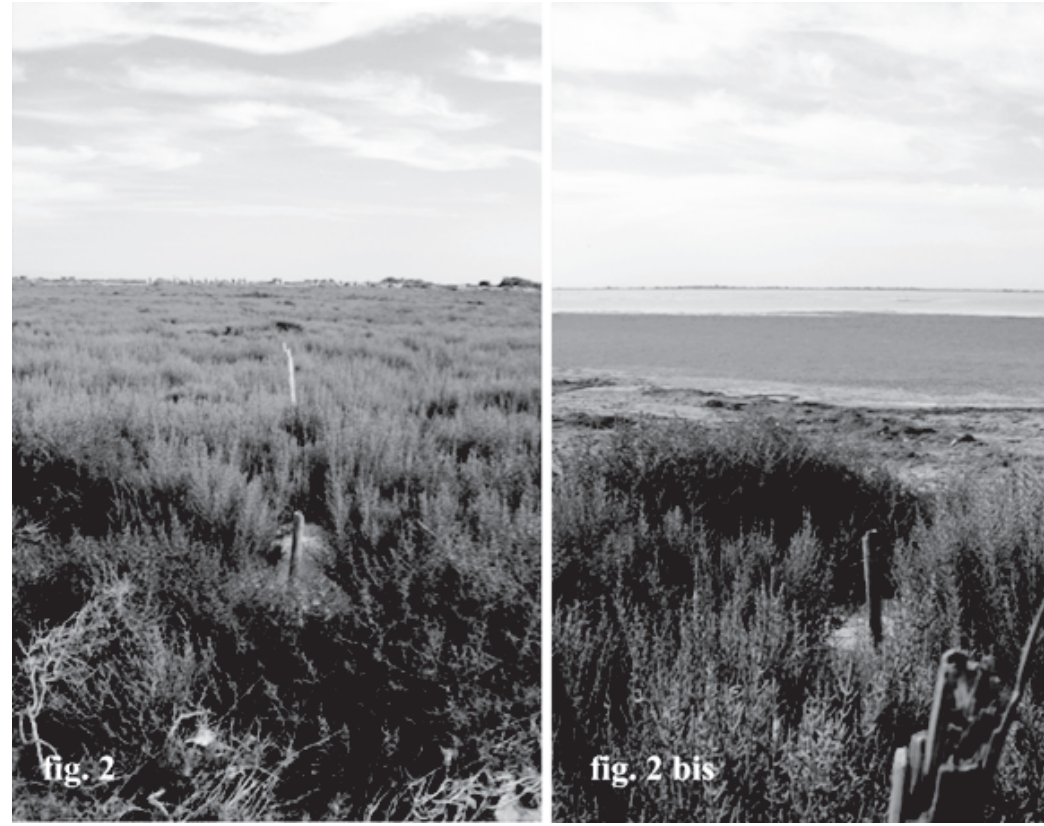

La végétation abondante qui entoure les piquets conduit le pêcheur à nourrir l'interprétation selon laquelle les étangs se retirent ou s'assèchent par faute de niveaux d'eau durablement suffisants.

De la même façon, des indications visuelles aident cet usager à produire une approximation de la baisse du niveau moyen («moyen», cette fois, et non pas «normal») à partir de l'étalement $\mathrm{du}$ "couvert hydraulique»; une correspondance mentale s'opère entre plusieurs mètres étalés horizontalement sur les "bords" de l'étang et l'extrapolation de quelques centimètres verticaux définissant la cote au centre de l'étang (voir la figure 3). 
Figure 3:

Repère visuel d'étalement de la masse d'eau pour évaluer une cote approximative

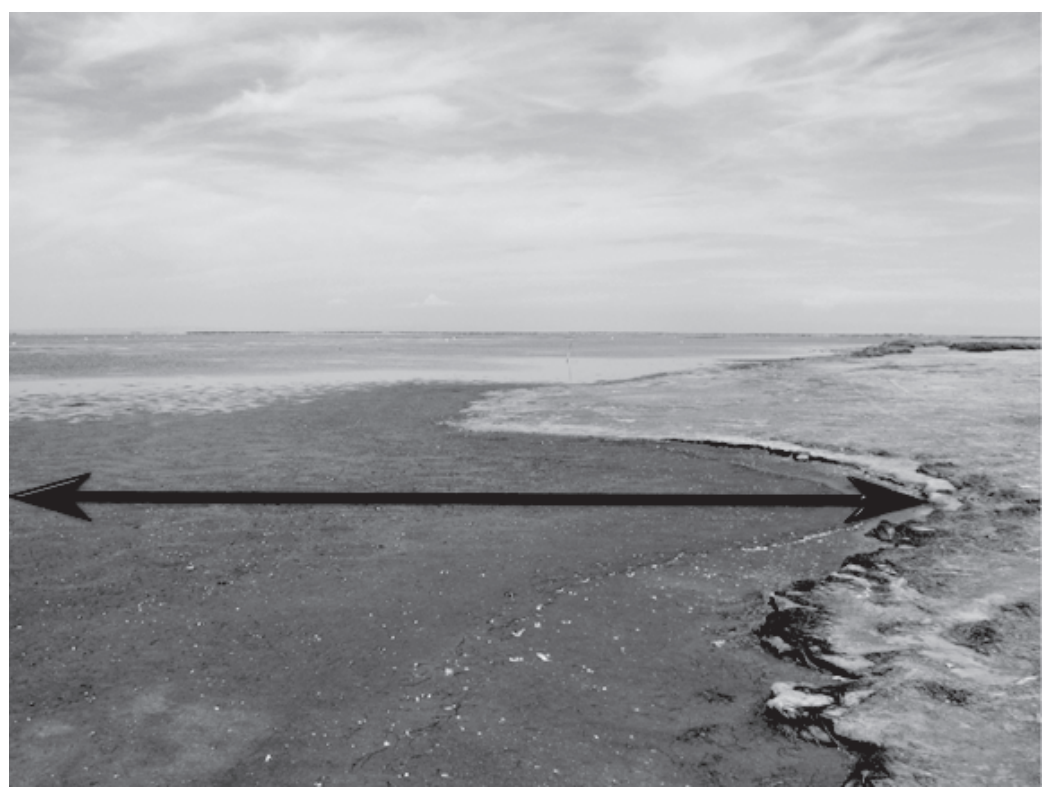

Cette visualisation s'accompagne d'une attention portée à la croûte de sable durcie, laissée par les temps de mise en eau prolongée et le battement des eaux. La hauteur de ces densifications de sable laisse également projeter une hauteur d'eau fictive, indiquant que l'étang, en temps normal, s'étale jusqu'à ce point (voir la figure 4). 
Figure 4:

Densifications du sable laissées par le recul de l'eau indiquant d'anciens niveaux de l'étang

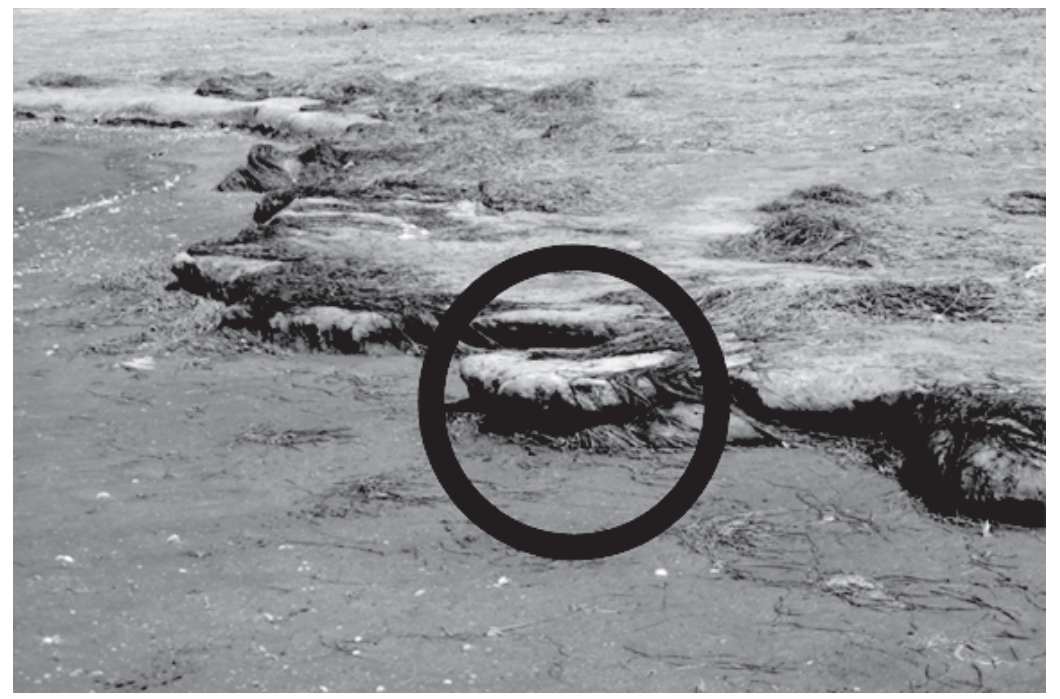

Indépendamment de la validité des interprétations que ce pêcheur fait des différents indices visuels, ceux-ci s'intègrent dans un ensemble de raisonnements. Mais ces raisonnements ne peuvent pas être pris en compte par l'espace de concertation tant que la prétention à l'objectivité de ces mêmes indices n'est pas reconnue par les membres de la CEDE.

Il en est ainsi de raisonnements liés à l'effet de rétention d'eau par certaines «baisses» qui, s’approfondissant au fil des assèchements successifs, vont, une fois remises en eau par coup de mistral, stocker une grande quantité d'eau dont elles priveront l'étang. D'autres raisonnements expliquent la réduction du niveau des étangs par un exhaussement des fonds causé par le développement rapide de certaines végétations aquatiques. Celles-ci seraient favorisées par une mauvaise gestion de l'eau et par des intrants agricoles et retiendraient à leur base des sables et des alluvions, comblant ainsi progressivement les étangs. 
Il semble donc que le manque d'intercompréhension au sein de la CEDE tient en partie à l'usage de référentiels sensibles et perceptuels très différents des courbes de moyennes des mesures du niveau du Vaccarès. Ces indices perceptuels et les raisonnements qui s'appuient sur eux échouent à s'inscrire dans le travail collectif d'objectivation du territoire nécessité par la gestion du pertuis à la mer. On peut ainsi se demander si l'objectivation accomplie dans la gestion collective ne peine pas, dans certains cas, à intégrer les représentations, les connaissances, les observations et le raisonnement qui offrent une forte dimension contextuelle et une spatialisation importante, renvoyant aux modes d'expression et de représentation du territoire vécu et pratiqué par les usagers et habitants.

\section{Lapprentissage organisationnel et l'objectivation du territoire}

L'inscription des représentations issues de la pratique du territoire dans le travail collectif d'objectivation se confronte à d'autres caractéristiques de la gestion concertée. Dans ce qui précède, l'objectivation du territoire se développait dans le plan de l'échange immédiat entre acteurs, tout en prenant appui sur des mesures et des dispositifs techniques définissant l'état moyen des étangs centraux. Néanmoins, l'objectivation du territoire dans la gouvernance ne peut pas être analysée à partir des seules relations épistémiques entretenues par les acteurs. En effet, pour être pérenne, la division du travail cognitif doit déboucher sur la formation de règles de décision et d'interprétation qui par leur institutionnalisation rendent les connaissances d'arrière-plan et les consensus collectifs accessibles à tout nouvel acteur sans qu'il lui soit nécessaire de les réexaminer ou de les reconstruire à chaque réunion ou à chaque décision.

C'est pourquoi, au sein d'un groupe ou d'une institution, un raisonnement collectif, une connaissance partagée ou un consensus d'interprétation vise, sous la forme d'acquis qui ne sont plus à discuter, une concrétisation organisationnelle qui l'autonomise des échanges entre les acteurs. 
En effet, de nombreux travaux approchent l'apprentissage collectif comme une forme d'institutionnalité de la raison dont le vecteur est l'exigence de coordination de l'action collective. Les travaux de l'économie des conventions, et singulièrement ceux d'Olivier Favereau qui comprend la norme (institutionnelle) comme un dispositif cognitif collectif ${ }^{46}$, vont en ce sens et convergent sur ce point avec la théorie de l'apprentissage organisationnel ${ }^{47}$. L'émergence de routines au cours d'interactions, la formulation de règles de décision, la constitution de procédures sont autant de formes d'établissement collectif d'apprentissages permettant de les rendre accessibles à de nouveaux acteurs ${ }^{48}$. On retrouve ici la conception de John Rawls, pour qui la règle fonctionne comme une théorie résumée, un condensé d'apprentissages et de décisions antérieures ${ }^{49}$, un acquis organisationnel permettant aux individus de remplacer un coûteux «savoirpourquoi" par un «savoir-comment $»^{50}$. En ce sens, la règle de décision marque un arrêt du travail collectif d'objectivation. De plus, le recours à des règles ou à des routines reconnues dans le groupe affranchit partiellement les acteurs de l'obligation de faire la démonstration de leur autorité épistémique pour justifier une décision dérivée de la règle.

Dans le cadre de la gestion participative d'un territoire, une règle de gestion est l'aboutissement d'un travail d'objectivation et vise, précisément, à ne plus rendre nécessaire ce travail lors de la gestion courante. On peut alors se demander si l'instauration de règles de gestion ne peut pas tacitement obéir à une logique contraire à celle de l'expression des représentations et des

$\overline{46}$ Olivier Favereau, «Organisation et apprentissage collectif : un paradigme non standard pour trois théories hétérodoxes», dans André Orléans (dir.), Analyse économique des conventions, Paris, Presses universitaires de France, 1994, p. 113-137.

47 Chris Argyris et Donald Schön, Apprentissage organisationnel. Théorie, méthode, pratique, Bruxelles, De Boeck, 2002.

48 Aaron Cicourel, op. cit.

49 John Rawls, "Two Concepts of Rules», Philosophical Review, vol. 64, n 1 , 1955.

50 Jean de Munck, L'institution sociale de l'esprit. Nouvelles approches de la raison, Paris, Presses universitaires de France, 1999, p. 138. 
connaissances singulières des habitants et usagers. En effet les règles collectives s'apparentant à des "résumés sélectifs ${ }^{51}$ ", leur construction tend à procéder par abstraction contextuelle, en réduisant la diversité des singularités territoriales et en condensant la profusion des expériences du territoire pratiqué. De ce point de vue, ce ne sera pas tant le caractère subjectif, reproductible, communicable d'une connaissance vernaculaire ou des représentations d'une catégorie d'usagers qui déterminera leur inscription ou leur non-inscription dans la compréhension collective du territoire. Ce seront davantage les logiques de contextualisation et de décontextualisation des données territoriales auxquelles ces règles de gestion doivent obéir pour être pleinement opératoires qui joueront sur la possibilité d'une connaissance donnée d'être collectivement retenue.

4.1. La quantité de choses que l'on a besoin d'ignorer pour gérer ou la naissance d'une règle de gestion

La gestion de l'eau en Camargue a longtemps obéi à une règle d'usage que l'on trouve mentionnée dans différentes conventions collectives $^{52}$, il s'agit de la règle des «trois vingt». Cette règle de gestion préconise que le niveau du Vaccarès doit varier entre -0,20 $\mathrm{m}$. NGF et $+0,20 \mathrm{~m}$. NGF et que la salinité de l'étang doit avoisiner la teneur de $20 \mathrm{~g} / \mathrm{l}$. La règle des «trois vingt» matérialise un accord social, un compromis apaisé entre les usages. Une salinité de $20 \mathrm{~g} / \mathrm{l}$ est supposée être favorable à la pêche et à la conservation de la nature, alors que la variation entre la cote inférieure et la cote supérieure garantirait la pérennité des cultures dont l'assolement se fait en automne. La règle des "trois vingt" met en accord les rejets massifs d'eau de la riziculture dans le Vaccarès, défavorables aux espèces piscicoles si la salinité s'en trouve trop fortement diminuée, et les conditions d'assolement pour l'agriculture (non poldérisée) qui imposent au Vaccarès des cotes assez basses.

51 Olivier Favereau, op. cit.; Jean de Munck, op. cit.

52 Notamment l'ancienne convention liant le syndicat de la digue-à-la-mer sur laquelle est situé le pertuis de la Fourcade et la commune des Saintes-Maries-de-la-Mer. 
Cette règle de gestion a le plus souvent été traitée comme une convention générale, tout en connaissant de nombreux aménagements contextuels ${ }^{53}$.

Par la suite, l'élévation constante du niveau marin a conduit, dans la gestion quotidienne du pertuis, à se départir de cette référence. Depuis les années 1980, le niveau de la mer s'est élevé de $0,10 \mathrm{~m}^{54}$, sans que l'étang du Vaccarès suive la même évolution, ce qui rend cette règle difficilement applicable. Pourtant la règle des «trois vingt» reste une référence occasionnelle parmi les acteurs qui participent à la gestion. Cela s'explique par le fait qu' elle fournit une convention de gestion permettant d'articuler les différentes conceptions sociales du territoire. Elle parvient à prendre sens dans les pratiques du territoire, en se situant dans la quotidienneté des usages, tout en offrant la représentation, attestée par la mémoire collective, d'un lien fonctionnel entre les différentes activités du territoire qui, s'il est respecté, permet d'éviter les situations de crise. Cette règle de gestion a donc, pendant longtemps, fourni un cadre d'objectivation où différentes représentations issues des territoires "vécus» pouvaient rencontrer l'approche technique et scientifique du territoire de projet. La révision de cette règle de gestion constitue un enjeu essentiel pour la problématique qui nous occupe.

Si les conditions hydrologiques actuelles rendent aujourd'hui impossible l'application de la règle des «trois vingt», les modalités de décision collective en conservent néanmoins le schème logique. Celui-ci consiste à décider de l'ouverture du pertuis à la mer en fonction de la cote atteinte par la mer. Lorsque la mer atteint une cote-limite donnée, un nombre correspondant de vannes doivent être ouvertes (ou fermées lorsque la cote est négative). La révision de ce schème logique implique donc un

\footnotetext{
53 Les archives de la CEDE montrent qu'elle devient, à partir de 1997, une sorte de règle des "deux quinze» : $-0,15 \mathrm{~m}$ NGF comme niveau minimal et une salinité de $15 \mathrm{~g} / \mathrm{l}$.

54 François Sabatier, «Modélisation de l'impact du changement climatique sur l'érosion des dunes. Cas pilote du nord du golfe de Beauduc (Camargue)", dans André Monaco et Wolfgang Ludwig (dir.), La zone atelier ORME, Versailles, Éditions QUAE, 2009, p. 315-322.
} 
changement profond. Le dépassement collectif de la règle des "trois vingt» se comprend ainsi comme un apprentissage institutionnel et organisationnel, résidant dans la production d'un nouveau principe de décision. Plus que de changer les décisions en les ajustant à l'évolution du milieu, il s'agit de changer la façon de décider, ce qui constitue un méta-changement. La production d'une nouvelle règle de gestion se comprend comme la constitution d'un nouveau cadre d'objectivation supposé permettre la représentation des différents usagers du territoire.

Une question se pose alors. L'application concrète d'une règle de gestion se confronte de facto à l'expression des différents usages et représentations du territoire; charge aux acteurs de faire valoir leur point de vue pour ce faire, et ce, avec les difficultés que l'on a pu voir précédemment. Est-ce que, pour autant, la construction collective du principe logique de cette règle permet, à son tour, la prise en compte des contraintes singulières des différents usages du territoire et des représentations correspondantes? Les réponses à cette question sont, sans nul doute, complexes et nombreuses. Aussi s'agira-t-il ici de simplement contribuer à cette problématique dans les limites de ce que l'étude de cas rend analysable. À ce titre, l'étude du dépassement par la CEDE de la règle des "trois vingt" suggère que le processus de construction d'une nouvelle règle de gestion obéit à un principe de réduction de la complexité des données et des facteurs entrant dans la gestion. Cette logique d'élaboration de la règle de gestion est potentiellement contraire à l'intégration de la diversité des représentations et des contextes des usagers. Se pose alors la question de l'articulation de ces deux niveaux d'objectivation du territoire.

Pour porter cette interrogation, il est utile de décrire comment naît in vivo une règle de décision concernant la gestion du pertuis de la Fourcade, gestion dont dépendent, dans de grandes proportions, la physionomie, les fonctionnalités et les usages du territoire rhodanien.

Le retour, en 2011, au sein de la CEDE du directeur adjoint du PNRC, acteur ne partageant pas nécessairement les préconstruits du groupe en raison de sa longue absence et étant, de 
ce fait, en position d'entrevoir des innovations possibles a conduit à une évolution des modalités de gestion. Les préconisations de gestion adoptaient un principe logique proche de celui de la règle des "trois vingt»: bien que n'adoptant pas les valeurs (et leur caractère figé) de cette règle de gestion, le principe restait celui de déduire un nombre d'ouvertures de vannes à partir de niveaux minimum et maximum de l'étang du Vaccarès.

La réapparition de ce membre, associée à un changement perceptible du mode de présidence des réunions - en raison du remplacement du président de la CEDE par un confrère moins directif - conduit à des interactions marquées par plus de latéralité (apartés, interpellation de membre à membre, temps morts, digressions...). Dans ce cadre, la règle de décision en vient, presque incidemment, à être de nouveau discutée. Ainsi, lors de la commission du 24 février 2011, au détour d'un "tassement» de la réunion, l'expert hydrologue trouve l'espace pour inciter à reformuler les préconisations en termes de différentiel mer-étang. Selon lui, il conviendrait de ne plus raisonner en termes de cotes d'alerte selon la logique suivie par la règle des "trois vingt» (par exemple, ouvrir toutes les vannes lorsque l'étang atteint la cote de $0,20 \mathrm{~m}$. NGF ou de 0,15 m. NGF). Il faudrait gérer les échanges avec la mer de façon à ce que la différence de niveaux entre la mer et les étangs n'excède pas la valeur de dix centimètres $(0,10 \mathrm{~m}$. NGF), et ce, quelles que soient les cotes de la mer et de l'étang.

Cette avancée d'apparence minime est pourtant essentielle. Il ne s'agit plus de préconiser l'ouverture de $x$ vannes lorsque le Vaccarès est au niveau $n$, suivant la forme logique de la règle des "trois vingt», mais de préconiser l'ouverture de $x$ vannes tant que la différence entre la mer et le Vaccarès est de $\Delta h$. Si ce mode de raisonnement pouvait être partiellement présent dans certains esprits, il n'était toutefois pas encore formalisé et ne fournissait pas un principe d'évaluation disponible pour la décision collective.

La proposition de l'hydrologue n'est pas immédiatement adoptée par les acteurs. Ce ne sera qu'à l'initiative du directeur adjoint du PNRC qu'elle se verra réintroduite dans la prise de 
décision. Ceci tendrait à montrer, par ailleurs, que l'autorité épistémique (celle de l'hydrologue, en l'occurrence) n'est pas suffisante pour modifier le cadre collectif d'objectivation, une autorité institutionnelle doit parfois l'étayer ou la ratifier.

Comment la suggestion faite par le directeur adjoint de mettre en application la préconisation de l'expert en hydrologie va-t-elle se concrétiser en une règle de gestion? Pour répondre à cette question, il nous faut considérer la transcription de l'échange durant lequel émergent les prémices d'une nouvelle règle de gestion (voir l'encadré 2).

Encadré 2 :

Extrait de la réunion de la CEDE du 24 février 2011 (premier extrait)

1. CHARGE DE MISSION PNRC: soyons pragmatiques.

Commençons par le plus simple. En cas de vent de secteur sud, niveaux marins hauts, on ferme tout? bon, ça, on continue?

[pas de réponse - consentement]

2. CHARGE DE MISSION PNRC: en cas de vent du nord? Que fait-on? Donc, là, je sais pas. Sachant qu'on a quand même des niveaux relativement bas.

[silence ]

3. CHARGE DE MISSION PNRC: que fait-on? On n'est pas dans une orientation à vouloir tout sortir

4. PRESIDENT DE LA COMMISSION GESTION DE L'EAU (CGO): je crois, on laisse 2

5. PRESIDENT DE SEANCE: on en laisse 2

[silence]

6. CHARGE DE MISSION PNRC: on laisse 3?

7. PRESIDENT DE SEANCE: ouais

8. DIR. ADJOINT PNRC: ben, quand tu dis vent du nord ça dépend de la force du vent!

9. CHARGE DE MISSION PNRC: oui, oui ça veut dire quand y a basculement éventuellement de l'eau -

10. DIR. ADJOINT PNRC: ah, si y a basculement, oui-

11. CHARGE DE MISSION PNRC: avec les niveaux d'eau qu'on a là... 
12. PRESIDENT DE LA CGO: je sais pas ce qu'il va faire aujourd'hui, mais on bascule pas grand-chose, non? Parce que d'autant moins qu'il y a pas beaucoup d'eau

13. CHARGE DE MISSION PNRC: donc, on fait quoi?

14. CONSEILLER MUNICIPAL: secteur nord, ça veut dire mistral qui souffle fort, hein parce que -

15. CHARGE DE MISSION PNRC: ça veut dire qu'on n'ouvre pas tout, déjà. D’accord avec lui?

16. EXPERT HYDROLOGUE: ça dépend ouais - ça dépend après y a - la suite. Ça dépend si on est prêt à dire...

17. PRESIDENT DE LA CGO : c'est déjà assez compliqué le présent comme ça!

18. EXPERT HYDROLOGUE: non, je veux dire après, on -

19. DIR. ADJOINT PNRC: mais, est-ce qu'on peut pas parler comme [l'expert hydrologue] l'a proposé pour le différentiel mer-étang, est-ce qu'il faudrait pas parler du différentiel étangmer? C'est-à-dire que par vent du nord, c'est-à-dire on a un différentiel +40 d'un côté est-ce qu'on ouvre ou est-ce qu'on n'ouvre pas? c'est la question qui peut se poser. Si on a qu'un différentiel de dix entre étangs et mer en faveur des étangs, est-ce qu'on ouvre ou pas? voilà c'est ça la question. Parce que, quelle que soit la force du vent, on s'en fout de la force du vent.

20. CHARGE DE MISSION PNRC: oui c'est vrai.

[silence ]

21. EXPERT HYDROLOGUE: Il faut raisonner en terme de décotes et puis jusqu'à quand aussi, par exemple on est descendu à -10 et on est à mi-mai par exemple.

22. DIR. ADJOINT PNRC: oui, faut croiser le niveau moyen avec - d'accord.

Au commencement de l'échange, les membres de la commission ne se sont pas approprié la suggestion de l'hydrologue. Ils continuent de formuler leurs préconisations en suivant des procédures routinisées qui obéissent au schème usuel de la règle des «trois vingt». La cote de l'étang ayant été communiquée précédemment dans la réunion, les membres se contentent de vérifier que le vent est correctement orienté pour prescrire l'ouverture de vannes correspondant habituellement à cette cote (tours de parole 4 à 6 ).

Les membres de la CEDE ont, depuis plusieurs années, l'habitude de gérer ensemble l'ouverture du pertuis. De ce fait, ils n'en viennent pas à formuler ni à discuter les présupposés sur lesquels 
ils s'appuient pour prendre leur décision, laquelle se fait «naturellement». Mais le directeur adjoint, rejoignant cette commission qu'après de très nombreuses années d'absence, ne partage pas ces présupposés. Aussi ne forme-t-il pas de préconisation sur un mode "automatique». C'est pourquoi la direction du vent ne lui apparaît pas, contrairement au reste du groupe, comme une donnée suffisante pour prendre une décision (tour de parole 8). La force du vent est, selon lui, un facteur indispensable à connaître. Or c'est là une donnée relativement complexe ${ }^{55}$ dont le reste de la commission avait pris pour habitude de se passer. La règle de décision usuelle cesse alors d'être opérante devant la complexité amenée par ce «nouvel» acteur.

C'est très précisément cette situation qui permet l'émergence d'une nouvelle règle de décision, sur la base de la proposition faite par l'hydrologue. Cette innovation est rendue possible parce qu'elle est vue comme pouvant répondre à une situation complexe. En effet, formuler systématiquement la prise de décision en termes de différentiel mer-étang permet de traiter certains facteurs pour négligeables et de réduire ainsi le nombre de données à connaître pour prendre une décision. Le raisonnement par différentiel permet notamment de faire abstraction de la force du vent sans influer sur la justesse de la décision. Cette nouvelle règle représente également l'intérêt de faire abstraction de l'orientation du vent, le chargé de mission du PNRC résumera effectivement plus loin, avec enthousiasme : "que le vent soit du nord ou soit $d u$ sud, on réagit pareil». La formalisation d'une règle de gestion procède ainsi d'une logique de réduction de ce qu'il est nécessaire de discuter pour décider. Instituer un mode de raisonnement collectif permet de s'en remettre à une règle pour ne plus avoir à traiter de la complexité des situations singulières et des facteurs qui les définissent.

On voit en cela qu'une règle de gestion ne s'impose pas uniquement en raison de l'autorité épistémique ou institutionnelle des

55 C'est une donnée très variable dans une journée. De plus, l'estimation de la proportionnalité entre la force du vent et le débit de l'eau évacuée nécessite des modélisations qui seraient peut-être difficilement communicables et utilisables lors de réunions. 
acteurs. Pour émerger, elle doit apparaître comme permettant de problématiser une situation grâce à un nombre plus réduit de facteurs, sans perte d'informations pertinentes.

C'est pourquoi, suivant cette logique, on peut se demander si la prise en compte de la singularité des observations localisées des usagers n'est pas exclue de ce processus, puisqu'elle apporte des éléments de contextualisation supplémentaires.

L'analyse de la suite de l'échange suggère différents cas de figure. Un conseiller municipal fait en effet remarquer que la mer «sera souvent au-dessus» du seuil de $10 \mathrm{~cm}$, seuil défini par les linéaments de la règle en formation. Cette remarque, confirmée par d'autres membres, suscite alors une innovation supplémentaire de la part du directeur adjoint du PNRC (voir l'encadré 3) :

\section{Encadré 3 :}

Extrait de la réunion de la CEDE du 24 février 2011 (second extrait)

1. DIR. ADJOINT PNRC: ah ouais... ou alors dans ce cas-là, on
peut faire aussi un système progressif: en dessus de $10 \mathrm{~cm}: 1$
vanne et en dessous: 3 vannes
2. CHARGE DE MISSION PNRC: oui!
3. CONSEILLER MUNICIPAL: ah oui, c'est une...
4. CHARGE DE MISSION PNRC: Donc on fait entre 0 et 10
cm de différentiel 3 vannes, au-delà de $10 \mathrm{~cm}$ jusqu'à $20-25$,
je sais pas?
5. DIR. ADJOINT PNRC: oui après -
6. CHARGE DE MISSION PNRC: on fait une vanne?
[acquiescements généraux]
7. CHARGE DE MISSION PNRC: au-delà de combien on
ferme tout?
8. EXPERT HYDROLOGUE: si on dit 20 - 25 c'est le niveau
marin moyen. Mais bon sur l'année...
9. PRESIDENT DE LA CGO : 20 ! c'est bon...
10. DIR. ADJOINT PNRC: on y est arrivé!

On voit que la règle de gestion s'affine grâce à des observations apportées par des acteurs locaux. En ce sens, il y a bien une intégration possible des représentations et des connaissances des 
usagers et acteurs "profanes». Il s'ensuit une innovation - la mise en œuvre d'une progressivité des modalités d'ouverture du pertuis - apportant une finesse de gestion sans précédent (voir la figure 5).

Figure 5:

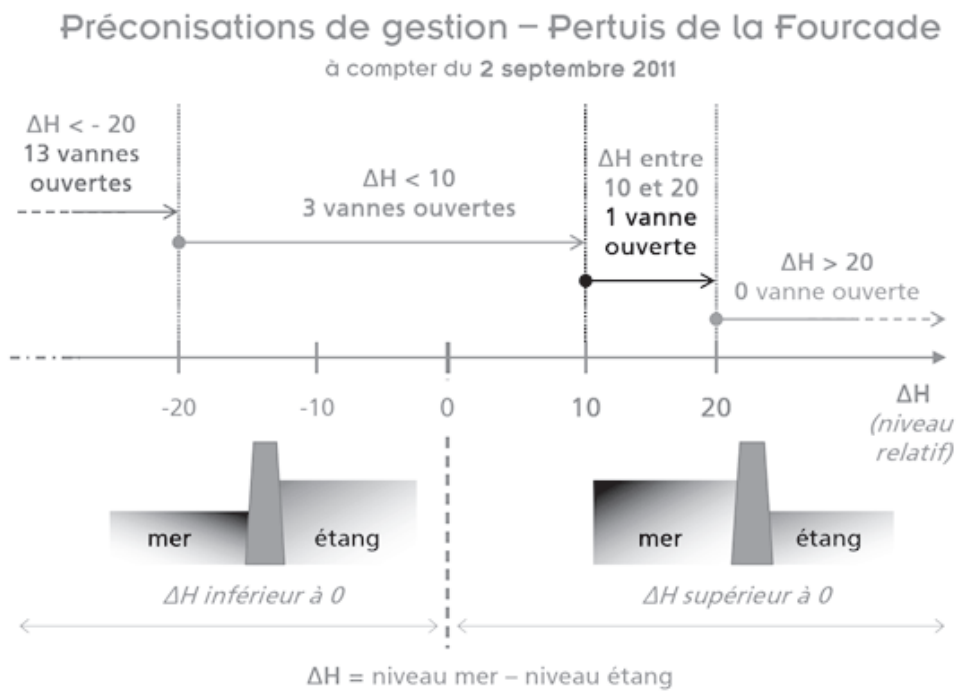

Schématisation du modèle de décision par différentiel mer-étang (schéma réalisé par Alain Dervieux pour la CEDE avec Marie Granier, chargée de mission PNRC).

La remarque de l'élu municipal a introduit un nouvel élément de complexité, obligeant un perfectionnement du raisonnement collectif. Mais cela apparaît comme un cas de figure particulier, remplissant des conditions spécifiques. En effet, l'intégration des observations de l'élu est possible parce que la commission était déjà parvenue à un nouveau principe logique de gestion. Dans une étape antérieure de la réflexion, qui visait la réduction de la complexité, la remarque de l'élu municipal aurait bloqué l'effort collectif de conceptualisation. Or la règle de gestion ayant déjà été mise à l'épreuve par la commission et appropriée par ses membres, l'observation de l'élu conduit à l'affinement de la règle plutôt qu'à sa remise en cause. L'observation de cet acteur trouve place dans la réflexion collective parce qu'elle est traduisible 
comme une gradation à l'intérieur du même principe logique adopté par la règle. Elle n'oblige pas à formuler un nouveau principe ni à aménager le principe retenu, mais simplement à le moduler. Il n'est pas certain qu'une observation ne remplissant pas ces conditions aurait conduit au même succès.

De plus, si l'innovation conceptuelle et cognitive en matière de règle de gestion est certaine, elle se fait principalement à la faveur d'assouplissements du cadre interactionnel (interventions très rares du président de séance) marqué par un mode d'interaction informel et l'arrivée d'un nouveau membre. Les facteurs ainsi réunis auraient très bien pu ne jamais l'être, cette innovation prend, sous cet angle, un aspect contingent. Dans ces circonstances, l'intégration de la diversité des représentations et des connaissances issues du territoire paraît difficilement pouvoir être formalisée en un objectif institutionnel, dans la mesure où les modalités de production des règles de gestion passent, dans le cas qui nous occupe, par des aspects contingents qui ne se décident pas. De ce fait, la contribution des usagers et des habitants à l'innovation de règle de gestion ne peut pas s'opérer sur le mode d'une consultation et plus difficilement encore suivant des modes formels que sont par exemple la délibération ou le vote.

La participation des acteurs non gestionnaires et non experts dépend, dans ce cas précis, de leur capacité à participer à un mode d'échange épistémique particulier, dont l'une des contraintes est de ne pas «importer» dans la conversation une quantité trop importante de détails circonstanciés ou de complexification. La réintroduction de considérations contextuelles et complexes ne peut se faire que progressivement, itérativement, à mesure que d'autres facteurs sont éliminés. Par ailleurs, la complexification de la règle avec la prise en compte d'observations contextuelles s'opère dans les limites que pose le souci de disposer d'une règle aisément mémorisable. C'est ainsi que l'affinement de la règle se cantonne à des valeurs rondes $(10,20,-10$ et -20) pour seulement quatre options de gestion (voir la figure 5).

Il ne faudrait pas pour autant en induire que la gestion collective peut durablement faire abstraction de la complexité du territoire 
et de la singularité de ses sous-systèmes dont les habitants et les usagers sont d'importants représentants.

4.2. La règle rattrapée par la complexité du territoire : contextualisation de la règle de gestion et déplacement de la décision en dehors de l'espace de discussion

À la suite de l'échange analysé, il est proposé par le chargé de mission du PNRC de vérifier, dans les quinze jours, avec le gardedigue l'opérationnalité de cette nouvelle règle de gestion, de son efficacité et de sa facilité d'application. Ce dernier met en garde la commission. D'une part, ses horaires de travail, du fait qu'il partage son activité entre plusieurs ouvrages hydrauliques, ne lui garantissent pas toujours de pouvoir arriver à temps pour augmenter l'ouverture du pertuis lorsque le différentiel mer-étang dépasse $10 \mathrm{~cm}$, alors que les régimes de vent changent rapidement, parfois, d'heure en heure. D'autre part, une ouverture de l'ouvrage décidée dans l'après-midi en fonction d'un différentiel peut s'avérer préjudiciable si les conditions hydrauliques et éoliennes s'inversent durant la nuit, aucune surveillance n'étant alors exercée. Une gestion adoptant des paramètres trop fins pourrait, dans ces conditions, être illusoire, voire dommageable.

Le souci du lien entre les préconisations de gestion et leur application exacte et rigoureuse est un point central dans l'activité de la CEDE. Là également, les dimensions cognitives de la gestion concertée sont cruciales, au point de pouvoir partiellement remettre en cause les efforts d'une «bonne» gestion collective. La discussion collective se heurte à la contrainte d'indexicalité des énoncés ${ }^{56}$, c'est-à-dire ici à l'impossibilité de décrire complètement le contexte d'application d'une préconisation et les facteurs entrant dans son application réelle. Si d'un point de vue cognitif et épistémique, la production de solutions de gestion nécessite une réduction des données mobilisées dans la discussion ainsi que l'abstraction de la pluralité des contextes, passé cette étape, la recontextualisation de la réflexion collective se confronte à la mise en œuvre des décisions. Même si les acteurs tentent de

56 Harold Garfinkel, op. cit. 
définir le mieux possible les conditions d'application des préconisations, le contexte "excède» toujours sa conceptualisation ou sa description. Anticiper et décrire exhaustivement toutes les données, tous les cas de figure, tous les facteurs que le garde-digue devra intégrer est simplement impossible.

Aussi ce garde-digue doit-il composer, par lui-même, avec de nombreuses données qui lui font "adapter» la préconisation. Les comptes-rendus que le garde-digue adresse au chargé de mission du PNRC apportent quelques exemples de ses motifs d'intervention et du non-respect occasionnel des préconisations faites par la CEDE. On peut notamment mentionner l'anticipation d'une «mer en formation» qui oblige le garde-digue à fermer les vannes prématurément afin d'anticiper sur le moment où il ne sera plus en service. Le garde-digue évoque également des ouvertures faites sur ordre de la mairie pour répondre à la demande des pêcheurs, sans que ces décisions ne fassent l'objet d'une consultation des représentants de la CEDE. D'autres fois, il justifie l'adaptation des préconisations le CEDE par l'évaluation du niveau des rejets rizicoles qu’il juge élevés pour la saison. S'ajoute à cela, la nécessite d'augmenter la durée d'ouverture du pertuis lorsque des poissons en grand nombre viennent "taper» aux vannes pour rejoindre la mer, sous le regard interloqué de touristes ou d'habitants qui comprennent mal qu'un Parc naturel incite à maintenir "captifs" des poissons dans quelques centimètres d'eau. Il y a également les fréquentes manœuvres illicites de vannes lorsque des usagers considèrent de leur propre chef que les échanges mer-lagune devraient être plus importants (ce sont parfois aussi des braconniers qui se spécialisent dans le commerce illicite de civelles).

Alors que différents membres de la CEDE traitent ces adaptations de la préconisation comme des imperfections de la gestion, il semble plutôt que l'action du garde-digue permette la réduction des données à mobiliser et à discuter collectivement pour pouvoir collectivement s'entendre. Ou, plus exactement, la décision collective est rendue possible par l'interprétation opérée quotidiennement par le garde-digue. D'une certaine façon, la capacité 
d'évaluation, d'initiative et d'interprétation du garde-digue permet d'adapter la préconisation au contexte en recréant en quelque sorte son indexicalité, c'est-à-dire en complétant la liste non exhaustive des conditions d'application de la décision collective. L'activité pratique et routinisée du garde-digue permet de placer en dehors du champ de la discussion des données qui, par leur grand nombre, handicaperaient le déroulement «fluide» de la décision collective. La figure 6, que nous avons réalisée sur la base des archives de la CEDE disponibles au départ de l'enquête, rend compte du rôle des contextualisations et des adaptations des préconisations par le garde-digue sur une période de trois ans.

Figure 6:

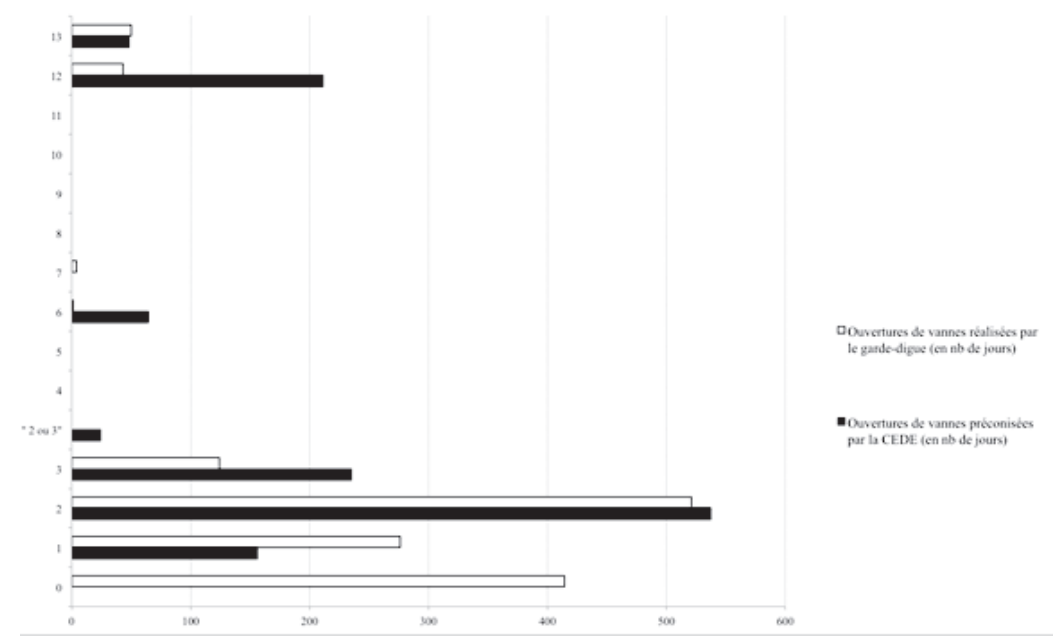

Préconisations d'ouverture et ouvertures effectives du pertuis de la Fourcade du 29-06-2006 au 31-12-2009. En ordonnées, la formulation de la préconisation par la CEDE, en abscisses, le nombre de jours où la préconisation référencée a été observée.

On observe clairement une adaptation opérée des préconisations au profit d'une tendance à la fermeture des vannes, associée à la sélection de valeurs non retenues initialement ( 0 et 7$)$. La gestion retrouve ici un ensemble de connaissances et de représentations issues d'une pratique particulière du territoire qui lui sont indispensables pour s'insérer dans la complexité matérielle 
et sociale de cet espace. La règle de gestion est bien un résumé sélectif d'apprentissages passés, comme une théorie condensée, mais cette simplification n'est rendue possible que parce que l'application de la règle de gestion est ajustée au contexte grâce à un ensemble de connaissances ancrées dans le territoire et dans sa pratique. Or cet ensemble complexe de facteurs qui rendent possible la règle se trouve sorti de l'espace de discussion. Cela pose la question des différents niveaux de gestion auxquels les représentations des usagers et des habitants peuvent apporter une contribution. Certes, la production de règles de gestion repose sur un principe de réduction des données et, en tant que telle, ne permet pas d'intégrer toute la diversité des facteurs contextuels. Néanmoins, on peut penser que les applications successives d'une règle de gestion entretient une sorte de casuistique ${ }^{57} \mathrm{de}$ celle-ci, cette casuistique s'opérant grâce à l'inscription des connaissances vernaculaires au sein du processus d'objectivation du territoire et à la diversification des points de vue exprimés. Le temps de la prise de décision n'est alors peut-être pas le seul, ni même le principal, instant du processus d'objectivation où doit s'opérer l'intégration des connaissances et des représentations issues des territoires vécus.

\section{Conclusion}

Létude de cas traité ne prétend pas à une représentativité des nombreuses situations de gestion participative d'un territoire. Elle fournit toutefois un matériau heuristique pour la description de deux processus épistémiques et cognitifs qui nous paraissent pouvoir se présenter dans des situations variées où se rencontrent gestionnaires, scientifiques, habitants et usagers du territoire. Les acteurs gestionnaires suivent un projet de territoire défini dans des plans de gestion, des chartes, des missions publiques, etc. Pour agir en ce sens, ces acteurs doivent entreprendre une objec-

57 Nous renvoyons à Christelle Gramaglia («La mise en cause environnementale comme principe d'association. Casuistique des affaires de pollution de rivières : l'exemple des actions contentieuses de l'Association nationale de protection des eaux et rivières (ANPER-TOS)», Thèse de doctorat en sociologie, École des Mines, Paris, 2006). 
tivation du territoire à laquelle sont appelés à participer les habitants et les usagers, ce qu'ils font principalement à partir de leurs représentations et de leurs connaissances. Dans l'étude de cas, nous avons décrit comment la dynamique des relations épistémiques et les propriétés cognitives et organisationnelles de la prise de décision pouvaient contraindre l'expression des représentations et des connaissances des acteurs non experts et non gestionnaires. Le choix de limiter notre propos aux processus décrits ne signifie aucunement que ces processus doivent être pensés comme exclusifs de tout autre ni qu'ils soient déterministes. Par leur aspect élémentaire, ces processus paraissent transposables à de nombreuses autres situations, mais ils n'impliquent pas nécessairement un constat pessimiste ou définitif quant à la gestion participative du territoire.

D'autres approches de l'objectivation du territoire sont possibles, notamment celles qui mettent l'accent sur une lecture inscrite dans un temps long ou qui donnent une plus grande place aux liens que les différentes catégories d'acteurs entretiennent spécifiquement avec une espèce animale ou un élément physique du milieu. Il aurait été possible, par exemple, d'aborder la différence des représentations en présence en partant des déplacements des espèces intéressant respectivement les différentes catégories d'acteurs et de leur sensibilité aux évolutions du milieu. L'objectivation aurait également pu être abordée en partant des différentes opérations de mise en forme symbolique du réel, depuis le prélèvement de mesures jusqu'aux opérations d'aménagement du milieu, en passant par les différentes formes d'énonciation (scientifiques ou non). Toutefois, notre intérêt s'est porté sur les échanges cognitifs et épistémiques, bien qu'il soit évident que cette échelle d'analyse ne corresponde qu'à quelques lignes du tableau d'ensemble. Une erreur notable consisterait à traiter ce choix analytique comme suffisant en lui-même, car cela rendrait la réflexion aveugle à divers facteurs. En particulier, la prédominance donnée dans l'analyse aux échanges discursifs et aux interactions situées ne permet pas de saisir les effets "à bas bruits", comme, par exemple, l'effet exercé sur l'espace de 
concertation par la recomposition des pratiques agricoles avec l'évolution de la Politique Agricole Commune (PAC). En dépit de cela, l'approche adoptée dans cet article vise à éclairer une dimension de la gestion participative qui est le plus souvent présupposée plutôt que décrite.

\section{Bibliographie}

Abric, Jean-Claude, Pratiques sociales et représentations, Paris, Presses universitaires de France, 1994.

Akrich, Madeleine, Michel Callon et Bruno Latour (dir.), Sociologie de la traduction. Textes fondateurs, Paris, Presse des Mines, 2006.

Allouche, Aurélien, «Les relations épistémiques de confiance et d'autorité dans l'action collective et le militantisme. Exemple par la sociologie des conflits environnementaux", Implications Philosophiques, numéro spécial «Modernité et confiance», 2014 [en ligne] http://www.implicationsphilosophiques.org, site consulté en octobre 2014.

Allouche, Aurélien et Laurence Nicolas, "Droits liés à l'eau dans la Camargue insulaire: à la croisée de la gouvernance environnementale et de la gestion des risques", Regions \& Cohesion, vol.1, n 3, 2011, p. 67-92.

Argyris, Chris et Donald Schön, Apprentissage organisationnel. Théorie, méthode, pratique, Bruxelles, De Boeck, 2002.

Berger, Peter et Thomas Luckmann, La construction sociale de la Réalité, Paris, Armand Colin, 1996.

Béthemont, Jacques, "Le riz et la mise en valeur de la Camargue", Revue de géographie de Lyon, vol. 37, n 2, 1962, p. 153-206.

Bohman, James, «Réaliser la démocratie délibérative comme mode d'enquête: le pragmatisme, les faits sociaux et la théorie normative», Tracés, vol. 15, n' 2, 2008, p. 1-29.

Boudon, Raymond, L'art de se persuader des idées douteuses, fragiles ou fausses, Paris, Fayard, 1990.

Bouvier, Alban, «La dynamique des relations de confiance et d'autorité au sein de la démocratie dite "participative" et "délibérative" ", Revue européenne des sciences sociales, vol. XLV, $\mathrm{n}^{\circ}$ 136, 2007, p. 181-230. 
Bouvier, Alban, «Dimensions axiologique, épistémologique et cognitive de la délibération publique : analyse d'un exemple", Cahiers d'économie politique, $\mathrm{n}^{\circ} 47,2004$, p. 215-234.

Bouvier, Alban et Bernard Conein (dir.), L'épistémologie sociale : une théorie sociale de la connaissance, Éditions EHESS, Paris, 2007.

Callon, Michel, "Éléments pour une sociologie de la traduction: la domestication des coquilles St-Jacques et des marins pêcheurs dans la baie de St. Brieuc», L'Année Sociologique, vol. 36, 1986, p. 169-208.

Calow, Peter, «Towards a Definition of Functional Ecology», Functional Ecology, vol. 1, no 1, 1987, p. 57-61.

Cefaï, Daniel et Isaac Joseph (dir.), L'Héritage du pragmatisme. Conflits d'urbanité et épreuves de civisme, La Tour-d'Aigues, Editions de l'Aube, 2002.

Chateauraynaud, Francis, Argumenter dans un champ de forces. Essai de balistique sociologique, Paris, Pétra, 2011.

Chauvelon, Philippe et al., "Satellite Remote Sensing and GIS Used to Quantify Water Input for Rice Cultivation (Rhône delta, France)», dans Manfred Owe et al. (dir.), Remote Sensing and Hydrology 2000, Wallingford, IAHS Publication, 2001, p. 446-450.

Cicourel, Aaron, Cognitive Sociology: Language and Meaning in Social Interaction, New York, Free Press, 1974.

Cicourel, Aaron, Le raisonnement médical. Une approche socio-cognitive, Paris, Seuil, 2002.

Dervieux, Alain, «La difficile gestion globale de l'eau en Camargue (France) : le Contrat de delta", Vertigo, 2005, [en ligne] http://vertigo. revues.org/2411, site consulté en octobre 2014.

Favereau, Olivier, «Organisation et apprentissage collectif : un paradigme non standard pour trois théories hétérodoxes», dans André Orléans (dir.), Analyse économique des conventions, Paris, Presses universitaires de France, 1994, p. 113-137.

Garfinkel, Harold, Studies in Ethnomethodology, Englewood Cliffs (NJ), Prentice-Hall, 1962.

Giddens, Anthony, Les conséquences de la modernité, Paris, L'Harmattan, 1994.

Gramaglia, Christelle, «La mise en cause environnementale comme principe d'association. Casuistique des affaires de pollution de rivières : l'exemple des actions contentieuses de l'Association nationale de protection des eaux et rivières (ANPER-TOS) ", Thèse de doctorat en sociologie, École des Mines, Paris, 2006.

Gras, Alain, Les macro-systèmes techniques, Paris, PUF, 1997. 
Grize, Jean-Blaise, Logique et langage, Paris, Ophrys, 1990.

Grize, Jean-Blaise, Pierre Vergès et Ahmed Silem, Les salariés face aux nouvelles technologies, Paris, Éditions du CNRS, 1987.

Guimelli, Christian, La pensée sociale, Paris, Presses universitaires de France, 1999.

Guimelli, Christian, Structures et transformations des représentations sociales, Lausanne, Delachaux et Niestlé, 1994.

Hardwig, John, «Epistemic Dependence», The Journal of Philosophy, vol. $82, \mathrm{n}^{\circ} 7,1985$, p. 335-349.

Hardwig, John, "The Role of Trust in Knowledge", The Journal of Philosophy, vol. 88, no 12, 1991, p. 693-708.

Heurteaux, Pierre, "Essai de quantification des termes du bilan hydrique des étangs du système Vaccarès Camargue (France)», Annales de Limnologie, vol. 30, n 2,1994, p. 131-144.

Kitcher, Philip, "The Division of Cognitive Labor », Journal of Philosophy, vol. 87, no 1, 1990, p. 5-22.

Lajarge, Romain, "Territorialisation(s) et parcs naturels régionaux", dans Alain Faure et Emmanuel Négrier (dir.), Les politiques publiques à l'épreuve de l'action locale. Critiques de la territorialisation, Paris, L'Harmattan, 2007, p. 69-78.

Latour, Bruno et Steve Woolgar, La vie de laboratoire, la production des faits scientifiques, Paris, La Découverte, 1988.

Lazega, Emmanuel, « «Pertinence et structure», Swiss Jounal of Sociology, vol. 37, n ${ }^{\circ}$ 1, 2011, p. 127-149.

Luhmann, Niklas, La confiance. Un mécanisme de réduction de la complexité sociale, Paris, Economica, 2006.

Mathevet, Raphaël, Camargue incertaine. Sciences, usages et natures, Paris, Buchet Chastel, 2004.

Mathevet, Raphaël et al., "Water Management in the Camargue Biosphere Reserve: Insights from Comparative Mental Models Analysis», Ecology and Society, vol. 16, $\mathrm{n}^{\circ}$ 1, 2011, [en ligne] http://www.ecologyandsociety. org/vol16/iss1/art43/, site consulté en octobre 2014.

Munck, Jean de, Linstitution sociale de l'esprit. Nouvelles approches de la raison, Paris, Presses universitaires de France, 1999.

Nachi, Mohamed, Introduction à la sociologie pragmatique. Vers un nouveau «style» sociologique?, Paris, Armand Colin, 2006.

Origgi, Gloria et Marta Spranzi, "La construction de la confiance dans l'entretien médical», dans Thierry Martin et Pierre-Yves Quiviger (dir.), Action médicale et confiance, Besançon, Presses universitaires de Franche-Comté, 2007, p. 227-246. 
Picon, Bernard, L'espace et le temps en Camargue, Arles, Actes Sud, 1978.

Rawls, John, «Two Concepts of Rules», Philosophical Review, vol. 64, ${ }^{\circ}$ 1, 1955.

Sabatier, François, «Modélisation de l'impact du changement climatique sur l'érosion des dunes. Cas pilote du nord du golfe de Beauduc (Camargue)", dans André Monaco et Wolfgang Ludwig (dir.), La zone atelier ORME, Versailles, Éditions QUAE, 2009, p. 315-322.

Schutz, Alfred, Le chercheur et le quotidien: phénoménologie des sciences sociales, Paris, Méridiens Klincksieck, 1987.

Searle, John, La construction de la réalité sociale, Paris, Gallimard, 1998.

Simmel, Georg, Secret et sociétés secrètes, Belval, Circé, coll. «Poche», 1991 [1906].

Strauss, Anselm, Miroirs et masques. Une introduction à l'interactionnisme, Paris, Métailié, 1992.

Vergès, Pierre «Représentations sociales de l'économie : une forme de connaissance», dans Denise Jodelet (dir.), Les représentations sociales, Paris, Presses universitaires de France, 1989, p. 387-405.

Vergès, Pierre, «Représentations sociales partagées, périphériques, indifférentes, d'une minorité», Les Cahiers internationaux de psychologie sociale, $\mathrm{n}^{\circ} 28,1995$, p. 77-95.

Watzlawick, Paul, La réalité de la réalité, Paris, Seuil, 1978. 